\title{
Direct numerical simulation of gaseous mixing layers laden with multicomponent-liquid drops: liquid-specific effects
}

\author{
By PATRICK C. LE CLERCQ AND JOSETTE BELLAN \\ Jet Propulsion Laboratory, California Institute of Technology, Pasadena, CA 91109-8099, USA
}

(Received 19 July 2004 and in revised form 20 December 2004)

A representation of multicomponent-liquid (MC-liquid) composition as a linear combination of two single-Gamma probability distribution functions (PDFs) is used to describe a large number of MC-liquid drops evaporating in a gas flow. The PDF, called the double-Gamma PDF, depends on the molar mass. The gas-phase conservation equations are written in an Eulerian frame and the drops are described in a Lagrangian frame. Gas conservation equations for mass, momentum, species and energy are combined with differential conservation equations for the first four moments of the gascomposition PDF and coupled to the perfect gas equation of state. Source terms in all conservation equations account for the gas/drop interaction. The drop governing equations encompass differential conservation statements for position, mass, momentum, energy and four moments of the liquid-composition PDF. Simulations are performed for a three-dimensional mixing layer whose lower stream is initially laden with drops colder than the surrounding gas. Initial perturbations excite the layer to promote the double pairing of its four initial spanwise vortices to an ultimate vortex. During the layer evolution, the drops heat and evaporate. The results address the layer evolution, and the state of the gas and drops when layers reach a momentum-thickness maximum past the double vortex pairing. Of interest is the influence of the liquid composition on the development of the vortical features of the flow, on the vortical state reached after the second pairing, and on the gas temperature and composition. The MC-liquid simulations are initiated with a single-Gamma PDF composition so as to explore the development of the double-Gamma PDF. Examination of equivalent simulations with n-decane, diesel and three kerosenes as the liquid, permits assessment of the single-species versus the MC-liquid aspect, and of mixture composition specific effects. Global layer growth and global rotational characteristics are unaffected by liquid specificity; however, the global mixing is highly liquid-specific. Also liquidspecific is the evolution of the ensemble-averaged drop characteristics and of the volumetric averages representing the gas composition. Visualized rotational characteristics show that the small-scale vortical activity increases with increased fuel volatility, which is confirmed by analysis of the vorticity budgets. Homogeneous-planeaverage budgets of the vorticity and vorticity-magnitude equations indicate that the stretching and tilting, and momentum-source terms are responsible for the difference among simulations. For all MC liquids, the gas displays a high level of composition heterogeneity, which can directly be traced to the original PDF representing the MC-liquid composition. Under most conditions, the single-Gamma PDF develops into a double-Gamma PDF; however, the extent of this transformation, indicative of vapour condensation onto drops, is not readily parametrized by the liquid volatility, initial carrier-gas temperature or trace vapour in the initial gas. 


\section{Introduction}

With few exceptions, most fuels used in energy production are mixtures of a multitude of chemical species. Devices based on fuel injection in the form of sprays use exclusively such fuels, called multicomponent (MC); examples are gasoline, kerosene and diesel. Until recently, it has been proposed to model the composition of such a fuel as the sum of all its individual species (e.g. Law \& Law 1982; Megaridis \& Sirignano 1990; Zheng \& Lee 2002) or as the sum of a solvent and a solute (Harstad \& Bellan 1991), thus relying on discretizing the mixture. Because of the daunting task of accounting for hundreds of species, leading to computationally unfeasible calculations, complex fuels have not been simulated so far. Similar impediments have been encountered in simulating other drop-laden flows where the liquid is MC, such as agricultural, paint, household and medical sprays. Additional applications may encompass atmospheric dispersal of liquid drops. In most applications, different chemical species have roles that affect specific phenomena in which other species may not participate. For combustion processes, examples are vapour ignition, flame propagation, pollutant formation, corrosion and coking. For agricultural, paint, household and medical sprays it may be desired to have surface deposition only for some species, while others are meant to evaporate before drops reach the target. For atmospheric dispersal, nucleation of species onto the drops may differentially change, according to the drop position, the composition of the liquid being dispersed.

Recent models of MC hydrocarbon-mixture drops based on a statistical rather than deterministic species representation have raised the possibility of simulating the full complexity of MC-liquid sprays (Tamim \& Hallett 1995; Hallett 2000; Lippert \& Reitz 1997; Harstad, Le Clercq \& Bellan 2003; Arias-Zugasti \& Rosner 2003; Harstad \& Bellan 2004) and thus to enable differentiating among a myriad of species. The common feature of all these recent models is the utilization of continuous thermodynamics (CT), a theory developed by Gal-Or, Cullinan \& Galli (1975) and by Cotterman, Bender \& Prausnitz (1985). In CT, the chemical potential for a mixture containing numerous components is appropriately represented, and the Gibbs function is derived through molecular thermodynamic methods in terms of the probability distribution function (PDF) describing the mixture composition. The concepts are fundamental and independent of the physicochemical model chosen for the chemical potential. From a specified initial PDF, the evolution of the mixture is determined by the physics of the situation encapsulated into thermodynamic relationships and/or conservation equations. Although the composition PDF generally depends on many variables, it can be appropriately chosen to depend on one or a restricted number of variables that govern the phenomena under consideration. For example, it has been shown, with validation, that the single-Gamma PDF (SGPDF) depending on the molar mass, $m$, can represent an entire homologous species class of hydrocarbons (Cotterman et al. 1985; Whitson 1983; Rätzsch \& Kehlen 1983; Chou \& Prausnitz 1986); for other applications, such as the computation of atmospheric hydrocarbon partitioning, the composition PDF was chosen to depend uniquely on the normal boiling point of the species, $T_{b}$ (Harstad 2005). When there is liquid-to-vapour phase change, Cotterman et al. (1985) have shown that the same mathematical form of the PDF applies in the gas phase as in the liquid from where the vapour originates. The flexibility of the CT approach in terms of the PDF mathematical form and of the choice of the PDF variable makes it attractive for a variety of studies.

Using the SGPDF approach, Le Clercq \& Bellan (2004) derived a formulation for a large number of drops evaporating in a temporal mixing layer undergoing transition 
to turbulence. In this formulation, both the liquid of the drops and the vapour composition were statistically described, and this statistical description was coupled to the deterministic description of the continuity, momentum and energy equations. The emphasis of that study was on comparisons between simulations performed with diesel and with n-decane as a representative single-component (SC) liquid diesel surrogate. Simulations were performed at $20 \%$ and $50 \%$ initial mass loadings and low (i.e. $8 \%$ ) relative initial-temperature difference between the free stream and the drops. Results were compared both during the layer evolution and at achieved transitional states. Visualizations of dynamic and thermodynamic variables at transition showed that the details of MC layers differ from their SC counterpart. Because MC-liquid drops evaporated slower due to the higher saturation temperature of the heavier species, their interaction time with the flow was longer, which permitted the development of a more complex small-scale vorticity structure in the flow at transition, and the creation of regions of higher drop number density which also displayed more structure, particularly in high-strain regions. In the SC case, the molar mass of the vapour ('vapour' is here defined as the ensemble of species evolving from the drops through evaporation) was inherently constant and its spatial distribution followed that of the vapour mass fraction; however, for the $\mathrm{MC}$ liquid, evaporation led to the liquid mean molar mass increasing and the variance initially decreasing.

As drops were transported into regions of different gas compositions, condensation occurred, leading to an eventual increase of the liquid variance. This realistic condensation of some species coexisting with the evaporation of other species was captured with only two additional conservation equations compared to the SC situation. The species released from the drop contributed to increasing both the mean molar mass and the variance of the vapour composition. Visualization of the mean molar mass spatial distribution in streamwise planes at transition revealed that the lighter components accumulated in the lower stream, being released early during evaporation, before the drops were entrained in the layer; intermediary molar mass species resided in the interior of the layer because they were released after the drops were entrained and therefore participated in the mixing process resulting from the double vortex pairing; the heavier components, which were released later in the drop lifetime, resided in regions of high drop number density. Therefore, despite turbulence features, a segregation of the chemical species occurred based on the time of their release from the drops. It is this segregation, which is important in many processes (including combustion, spray dispensing and atmospheric dispersion), that cannot be captured by an SCliquid approximation.

Harstad et al. (2003) have shown that SGPDF models applied to drop evaporation (Tamim \& Hallett 1995; Hallett 2000; Lippert \& Reitz 1997) are, however, restricted to applications where the gas surrounding the drop contains only small quantities of vapour species mixed with a carrier gas (e.g. air). A combination of two Gamma PDFs, called the double-Gamma PDF (DGPDF), as a function of $m$ is necessary to capture the evaporation of drops in a gas containing substantial quantities of vapour (Harstad et al. 2003), as in strongly evaporating sprays. In these sprays, as drop heating is initiated, the lighter species evaporate first, and, as the drops move in the flow, it is these species condensation onto drops that gives rise to the second lower$m$ peak of the PDF. The versatility of the DGPDF approach has further been demonstrated by Harstad \& Bellan (2004) who have enlarged the DGPDF concept through appropriate thermodynamic modelling and shown that a single DGPDF can represent several homologous hydrocarbon species classes. 
This study generalizes the CT modelling approach of Le Clercq \& Bellan (2004) to a variety of fuels of practical interest as an example of complex liquids, and addresses the problem of MC-liquid drop evaporation in situations where evaporation could be more vigorous, thus requiring utilization of the DGPDF instead of the SGPDF employed by Le Clercq \& Bellan (2004). Having already studied the effect of the initial mass loading (Le Clercq \& Bellan 2004), the emphasis is here on elucidating those vortical aspects of the flow that are affected by the liquid composition, on the species spatial distribution as a function of the fuel characteristics, and on how this distribution might change with increasing relative initial-temperature difference between the free stream and the drops. All simulations are performed in the pre-transitional regime so as to uncouple the composition aspect from turbulence features of the flow. The interest is in exploring the influence that liquid specificity has on the flow/drop coupled interaction, from the standpoint of both flow and drop characteristics; comparisons with a typical SC liquid are also performed. Because the drop/flow interaction exists only as long as the drop has not entirely evaporated, the interaction characteristic time is influenced by the liquid volatility, the initial drop size, the initial drop temperature, the initial gas composition and the initial gas temperature, particularly compared to the liquid boiling temperature. Thus, at the same drop size and gas characteristics, SC-liquid drop evaporation is governed by the single value of the liquid boiling temperature (at the specified pressure), while for MC-liquid drops, the boiling temperature may continuously change with composition as the drop evaporates. In this respect, the usefulness of the liquid statistical representation of the composition is to allow the study of MC-liquid drop/flow interaction at higher relative initialtemperature difference between the free stream and the drops than is possible with SC liquids.

This investigation is conducted using the direct numerical simulation (DNS) methodology wherein all scales of the flow are resolved. This methodology was initially devised for single-phase flows and was extended by Boivin, Simonin \& Squires (1998) to two-phase flows with particles that are much smaller than the Kolmogorov scale and which have a volumetrically small loading $\left(\simeq 10^{-3}\right)$. Indeed, Boivin et al. (1998) showed that the drops can be treated as point sources of mass, momentum and energy from the gas-phase perspective, and thus it is then appropriate to perform simulations using a gas-phase resolution that is adequate for single-phase flow by following the gas phase in an Eulerian frame and tracking the drops in a Lagrangian frame. The terminology DNS, while not strictly accurate, is traditionally applied to such simulations, and several recent studies have used this DNS methodology (Boivin et al. 1998; Mashayek 1998a, b; Mashayek \& Jaberi 1999; Réveillon \& Vervisch 2000; Okong'o \& Bellan 2004).

This paper is organized as follows: In $\S 2$ the conservation equations are presented in the framework of the DGPDF CT formulation. Section 3 is devoted to the description of the configuration, the boundary and initial conditions, and then of the results. These results encompass global layer characteristics, flow visualizations, and homogeneousplane-average information. A summary and conclusions are offered in $\S 4$.

\section{Conservation equations}

The equations are derived under the assumption that the $\mathrm{MC}$ liquid and gas compositions are described by

$$
P\left(m ; \alpha_{1}, \beta_{1}, \alpha_{2}, \beta_{2}, \epsilon\right)=(1-\epsilon) f_{\Gamma}^{(1)}+\epsilon f_{\Gamma}^{(2)},
$$


where $f_{\Gamma}^{(k)}=f_{\Gamma}\left(m ; \alpha_{k}, \beta_{k}\right)$ with integer $k=1,2, \epsilon$ is a weighting parameter $(0 \leqslant \epsilon \leqslant 1)$, $\int_{\gamma}^{\infty} P(m) \mathrm{d} m=1$ and

$$
f_{\Gamma}(m)=\frac{(m-\gamma)^{\alpha-1}}{\beta^{\alpha} \Gamma(\alpha)} \exp \left[-\left(\frac{m-\gamma}{\beta}\right)\right]
$$

where $\Gamma(\alpha)$ is the Gamma function. The origin of $f$ is specified by $\gamma$, and its shape is determined by $\alpha$ and $\beta$. Thus, under the assumption that $\gamma_{1}=\gamma_{2}=\gamma$, at each time $t, P\left(m ; \alpha_{1}, \beta_{1}, \alpha_{2}, \beta_{2}, \epsilon\right)$ is determined by the vector $\eta \equiv\left(\alpha_{1}, \beta_{1}, \alpha_{2}, \beta_{2}, \epsilon\right)$. Harstad, Le Clercq \& Bellan (2003) have shown that $P$ can be determined by an inverse mapping from its first four moments, $\theta, \psi, \xi_{3}$ and $\xi_{4}$, with a fifth parameter empirically calculated. These moments are defined as

$$
\xi_{n l} \equiv \int_{\gamma}^{\infty} m^{n} P_{l}(m) \mathrm{d} m, \xi_{n v} \equiv \int_{\gamma}^{\infty} m^{n} P_{v}(m) \mathrm{d} m,
$$

for integer $n \geqslant 1$, where subscript $l$ denotes the liquid and subscript $v$ denotes the vapour. At each $t, P_{l}$ describes the liquid-fuel composition, which is specific for each drop, and $P_{v}$ describes the vapour composition, which varies with spatial location. Comparisons between the DGPDF and a discrete 32-species mixture results showed generally very good, and at most conditions excellent, agreement (Harstad et al. 2003).

\subsection{Gas-phase equations}

The generic Eulerian gas-phase conservation equations for continuity, momentum, energy, species and DGPDF first four moments $\left(\theta_{v}, \psi_{v}, \xi_{3 v}, \xi_{4 v}\right)$ representing the composition can be succinctly written as

$$
\frac{\partial \Phi}{\partial t}+\frac{\partial\left[\Phi u_{j}\right]}{\partial x_{j}}=\frac{\partial[\Psi(\Phi)]}{\partial x_{j}}+S,
$$

where

$$
\Phi=\left\{c, c m u_{i}, c m e_{t}, c X_{v}, c X_{v} \theta_{v}, c X_{v} \psi_{v}, c X_{v} \xi_{3 v}, c X_{v} \xi_{4 v}\right\}
$$

is the vector of the conservative variables,

$$
\begin{aligned}
\Psi(\Phi)= & \left\{c m \mathscr{D} \frac{\partial}{\partial x_{j}}\left[\frac{X_{v}}{m}\left(1-\frac{\theta_{v}}{m_{a}}\right)\right], p \delta_{i j}-\tau_{i j},-u_{i} \tau_{i j}-\lambda \frac{\partial T}{\partial x_{j}}+\sum_{\zeta=1}^{N} J_{D \zeta, j}\left(h_{\zeta}-h_{a}\right),\right. \\
& c m \mathscr{D} \frac{\partial}{\partial x_{j}}\left(\frac{X_{v}}{m}\right), c m \mathscr{D} \frac{\partial}{\partial x_{j}}\left(\frac{X_{v} \theta_{v}}{m}\right), c m \mathscr{D} \frac{\partial}{\partial x_{j}}\left(\frac{X_{v} \psi_{v}}{m}\right), c m \mathscr{D} \frac{\partial}{\partial x_{j}}\left(\frac{X_{v} \xi_{3 v}}{m}\right), \\
& \left.c m \mathscr{D} \frac{\partial}{\partial x_{j}}\left(\frac{X_{v} \xi_{4 v}}{m}\right)\right\}
\end{aligned}
$$

is the diffusional flux vector corresponding to $\Phi$, and

$$
S=\left\{S_{\text {mole }}, S_{\text {mom }, j}, S_{\text {en }}, S_{\text {mole }}, S_{\text {mass }}, S_{\psi}, S_{\xi_{3}}, S_{\xi_{4}}\right\}
$$

is the source term vector of components $S_{r}$ associated with $\Phi$. In (2.4)-(2.6) $c$ is the molar density, $x_{i}$ is the $i$ th spatial coordinate, $\boldsymbol{u}$ is the mass-averaged velocity, $X$ is the mole fraction, $m=\theta_{v} X_{v}+m_{a}\left(1-X_{v}\right)$ is the molar mass where $m_{a}$ is the carrier gas molar mass (subscript $a$ denotes the carrier gas), $\mathscr{D}$ is an effective diffusion coefficient defined by Harstad \& Bellan (2004) as the proportionality coefficient between the vapour mass flux and $\operatorname{cm} \nabla\left(X_{v} / m\right), p$ is the pressure, $\tau_{i j}$ is the viscous stress tensor, $\delta_{i j}$ is the Kronecker symbol, $e_{t}=e_{k}+e_{i n t}=u_{i} u_{i} / 2+h-p / \rho$ is the total energy of 
the gas, $\rho=m c$ is the mass density, $h$ is the enthalpy, $\lambda$ is the thermal conductivity and $T$ is the gas temperature. The last term in the flux of the energy equation is the portion of the heat flux due to transport of species by the molar fluxes. The source vector arises from the coupled interaction of drops and gas, and is presented in $\S 2.3$.

The differential conservation equations are coupled to the equation of state

$$
p=\left(\rho R_{u} T\right) / m=c R_{u} T
$$

where $R_{u}$ is the universal gas constant.

\subsection{Drop equations}

This formulation parallels that of Okong'o \& Bellan (2004) for SC-liquid drops, with the added complexity of modelling the liquid composition. Each drop is individually simulated, meaning that we do not resort to utilizing 'computational' drops as an approximation. The liquid mass density is assumed constant, although the liquid molar density may vary; also, the drop size is negligibly affected by liquid thermal-expansion effects, as verified a posteriori in $\$ 3.2 .2$. Under the assumptions of quasi-steady gas phase with respect to the liquid phase, justified by $\rho / \rho_{l}=O\left(10^{-3}\right)$ (Williams 1965), and of uniform internal drop properties, justified by the relatively small evaporation rate (Makino \& Law 1988; an equivalent form of their criterion is checked a posteriori in §3.2.2), the Lagrangian conservation equations for each drop position $\chi$, velocity $v$, energy, mass $M_{d}=V_{d} \rho_{l}=\pi \rho_{l} D^{3} / 6(V$ and $D$ are the drop volume and diameter, and subscript $d$ denotes the drop), and composition are

$$
\begin{aligned}
& \frac{\mathrm{d} \chi_{i}}{\mathrm{~d} t}=v_{i}, \quad \frac{\mathrm{d} v_{i}}{\mathrm{~d} t}=\frac{F_{i}}{M_{d}}, \\
& M_{d} C_{l} \frac{\mathrm{d} T_{d}}{\mathrm{~d} t}=\underbrace{\frac{\lambda N u}{D} \frac{\ln \left(1+B_{T}\right)}{B_{T}} A\left(T-T_{d}\right)}_{Q_{\text {conv-dif }}}+\underbrace{\dot{m}_{d} \frac{L_{v e f f}}{B}}_{Q_{\text {evap }}}, \\
& \dot{m}_{d} \equiv \frac{\mathrm{d} M_{d}}{\mathrm{~d} t}=-\frac{S h}{3 \mathrm{Sc}} \frac{M_{d}}{\tau_{d}} \ln (1+B), \\
& \frac{\mathrm{d} \xi_{n l}}{\mathrm{~d} t}=\frac{3 \theta_{l}}{2 B D^{2}} \frac{\mathrm{d} D^{2}}{\mathrm{~d} t}\left\{\left[\frac{X_{v}}{m}-\frac{X_{v}^{(s)}}{m^{(s)}}(1+B)\right] \xi_{n l}+\frac{X_{v}^{(s)} \xi_{n v}^{(s)}}{m^{(s)}}(1+B)-\frac{X_{v} \xi_{n v}}{m}\right\},
\end{aligned}
$$

for integer $n \in[1,4]$, where the gas phase at the drop location, interpolated from the Eulerian solution, acts as the drop far field. $F_{i}=\left(M_{d} / \tau_{d}\right) f_{1}\left(u_{i}-v_{i}\right)$ where $\tau_{d}=$ $\rho_{l} D^{2} /(18 \mu)$ is the particle time constant for Stokes flow, $T_{d}$ is the drop temperature, $C_{l}$ is the liquid heat capacity at constant pressure, $A=\pi D^{2}$ is the drop-surface area and $\mu$ is the viscosity of the carrier gas; $\operatorname{Pr}=\mu C_{p} /(\lambda m)$, where $C_{p}$ is the gas heat capacity at constant pressure, and $S c=\mu /(\rho \mathscr{D})$ are the Prandtl and the Schmidt numbers respectively. The Nusselt, $N u$, and the Sherwood, $S h$, numbers are semiempirically modified using the Ranz-Marshall correlations, accounting for convective heat and mass transfer effects (Miller \& Bellan 1999), with the similarity assumption $N u=2+0.552 \operatorname{Re}_{s l}^{1 / 2}(\operatorname{Pr})^{1 / 3}, S h=2+0.552 \operatorname{Re}_{s l}^{1 / 2}(S c)^{1 / 3}$. Also, $f_{1}$, given in Miller \& Bellan (1999),

$$
\begin{gathered}
f_{1}=\frac{1+0.0545 R e_{s l}+0.1 R e_{s l}^{1 / 2}\left(1-0.03 R e_{s l}\right)}{1+a\left|R e_{b}\right|^{b}}, \\
a=0.09+0.077 \exp \left(-0.4 R e_{s l}\right), \quad b=0.4+0.77 \exp \left(-0.04 R e_{s l}\right),
\end{gathered}
$$


is an empirical correction to Stokes drag accounting for both finite droplet Reynolds numbers using the slip Reynolds number $R e_{s l}=|\boldsymbol{u}-\boldsymbol{v}| \rho D / \mu$ where $(\boldsymbol{u}-\boldsymbol{v})$ is the slip velocity, and a Reynolds number $R e_{b}=U_{b} \rho d / \mu$ based on the evaporation blowing velocity $U_{b}$ obtained from the mass conservation relation at the drop surface, $\dot{m}_{d}=-\pi \rho D^{2} U_{b}$. The correlation of (2.13) is valid for the ranges $0 \leqslant R e_{s l} \leqslant 100$ and $0 \leqslant R e_{b} \leqslant 10 . B=\left(Y_{v}^{(s)}-Y_{v}\right) /\left(1-Y_{v}^{(s)}\right)$ is the Spalding number, where $Y_{v}=X_{v} \theta_{v} / m$ is the vapour mass fraction, $Y_{a}=1-Y_{v}$, and $\left(1+B_{T}\right)=(1+B)^{1 / L e}$ with $L e$ being the Lewis number, $L_{\text {veff }}$ is an effective latent heat (Harstad et al. 2003) whose expression is given in Appendix A, and the superscript $(s)$ denotes the drop surface. At this surface, the classical boundary conditions of temperature equality, and mass, species, momentum and energy flux conservation apply (Williams 1965) and under the ideal-mixture assumption, Raoult's law relates the drop and gas PDFs

$$
P_{v}^{(s)}=\frac{p_{a t m}}{X_{v}^{(s)} p} \exp \left[\frac{m L_{v}(m)}{R_{u} T_{b}(m)}\left(1-\frac{T_{b}(m)}{T_{d}}\right)\right] P_{l}^{(s)},
$$

where $p_{a t m}=1 \mathrm{~atm}$ and $L_{v}(\mathrm{~m})$ and $T_{b}(\mathrm{~m})$ are the latent heat and the normal boiling point, correlated as functions of $m$ by Harstad et al. (2003) using Trouton's law,

$$
\Delta s_{\lg }=m L_{v} / T_{b} \simeq 10.6 R_{u},
$$

and

$$
T_{b}(m)=A_{b}+B_{b} m,
$$

where constants $A_{b}$ and $B_{b}$ are specified in Appendix A.

\subsection{Source terms}

The source-term-vector components of (2.7) are

$$
\begin{aligned}
S_{r}= & -\sum_{q=1}^{N} \frac{w_{q}}{\Delta V_{q}}\left[\Lambda_{r}\right]_{q}, \\
\Lambda= & \left\{\frac{\mathrm{d} \mathscr{N}}{\mathrm{d} t}, F_{j}+\frac{d\left(\mathscr{N} \theta_{l}\right)}{\mathrm{d} t} v_{j}, v_{j} F_{j}+Q_{\text {conv-diff }}+Q_{\text {evap }}+\dot{m}_{d}\left(\frac{v_{j} v_{j}}{2}+C_{p l} T_{d}\right),\right. \\
& \left.\frac{\mathrm{d} \mathscr{N}}{\mathrm{d} t}, \frac{\mathrm{d}\left(\mathscr{N} \theta_{l}\right)}{\mathrm{d} t}, \frac{\mathrm{d}\left(\mathscr{N} \psi_{l}\right)}{\mathrm{d} t}, \frac{\mathrm{d}\left(\mathscr{N} \xi_{3 l}\right)}{\mathrm{d} t}, \frac{\mathrm{d}\left(\mathscr{N} \xi_{4 l}\right)}{\mathrm{d} t}\right\}
\end{aligned}
$$

where $\mathscr{N}=M_{d} / \theta_{l}$ is the number of moles in the drop, $N$ is the number of drops, and the summation in (2.18) is over all drops residing within a local numerical discretization volume, $\Delta V_{q}$. Following the SC methodology (Miller \& Bellan 1999; Okong'o \& Bellan 2004), a geometric weighting factor $w_{q}$ distributes the individual drop contributions to the nearest eight grid points in proportion to their distance from the drop location. Le Clercq \& Bellan (2004) explained that because convective effects dominate the species flux term, for MC flows differential species diffusivity is negligible in transport from the drop location to the grid nodes. Because numerical diffusion induced by distributing the Lagrangian source terms at the Eulerian nodes is proportional to the ratio of $V_{d}$ to the Eulerian mesh volume, this potential effect is here negligible since this ratio is initially $O\left(10^{-3}\right)$ (see discussion in $\S 3.1$ ) and decreases with $t$, as the drops evaporate. 


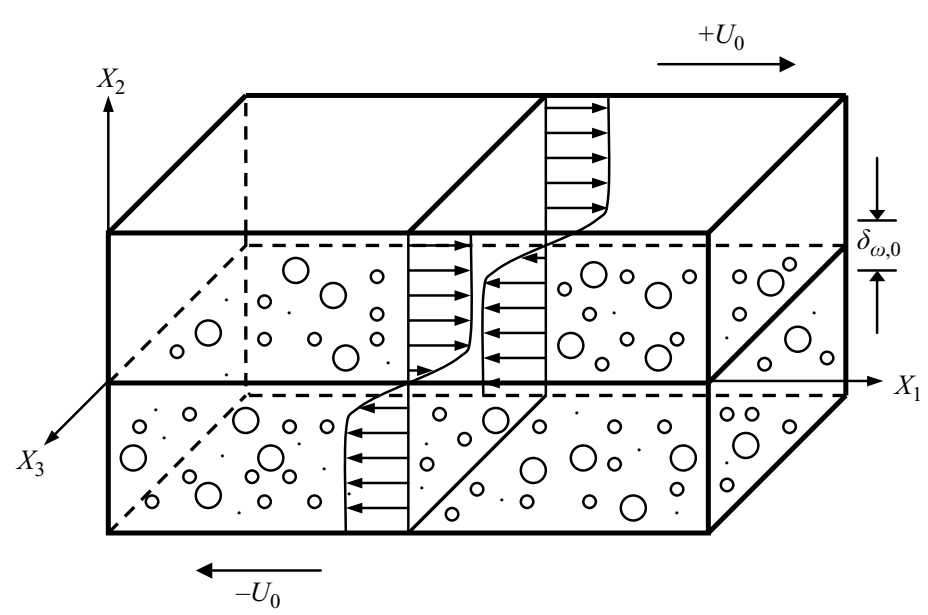

FIGURE 1. Mixing layer configuration.

\section{Results}

\subsection{Configuration, boundary and initial conditions, and numerics}

Figure 1 illustrates the mixing layer and shows the streamwise, $x_{1}$, cross-stream, $x_{2}$, and spanwise, $x_{3}$, coordinates with lengths $L_{1}=4 l_{1}=29.16 \delta_{\omega, 0}, L_{2}=1.1 L_{1}$, and $L_{3}=$ $4 l_{3}=0.6 L_{1}$, with $L_{1}=0.2 \mathrm{~m}$, where $l_{1}$ and $l_{3}$ are forcing wavelengths in the $x_{1}$ - and $x_{3}$-directions, which are used to perturb the layer so as to promote roll-up and pairing (Miller \& Bellan 1999; Moser \& Rogers 1991; Miller \& Bellan 2000). The perturbations used to excite the layer are described elsewhere (Moser \& Rogers 1991; Miller \& Bellan 1999); their relative amplitudes with respect to the circulations are $10 \%$ and $2.25 \%$ in the spanwise and streamwise directions, respectively. The evolution of the layer comprises two pairings of the four initial spanwise vortices to form a single vortex. $\delta_{\omega, 0}=\Delta U_{0} /\left\langle\partial u_{1} / \partial x_{2}\right\rangle$ is the initial vorticity thickness (subscript 0 denotes the initial condition) where $\Delta U_{0}=2 U_{0}$ is the velocity difference across the layer, the brackets \langle\rangle indicate averaging over homogeneous $\left(x_{1}, x_{3}\right)$-planes, and the initial mean streamwise velocity has an error-function profile (Miller \& Bellan 1999). The drops are randomly distributed throughout $x_{2}<0$ with uniform number density and uniform temperature $T_{d, 0}<T_{0}$, where $T_{0}$ is the initial, uniform, carrier gas temperature. Because $T_{d, 0}<T_{0}$, drop heating ensues past the initial condition, resulting in species evaporation. The mean drop number density profile is smoothed near the centreline, $x_{2}=0$, using an error function profile. Table 1 summarizes the initial conditions. The initial drop-slip velocity with respect to the gas is null. Comparing $\mathrm{MC}$ - and SC-liquid physical properties, it is obvious that one cannot initialize the calculation with the same $\rho_{l}, M L_{0}, D_{0}$ and $S t_{0}$, where $M L_{0}$ is the initial mass loading defined as the total initial mass of the liquid relative to the total mass of the gas in the laden stream and $S t=\tau_{d} \Delta U_{0} / \delta_{\omega, 0}$ is the Stokes number. Following the well-accepted methodology of comparing simulations performed with specified non-dimensional numbers rather than physical quantities (Batchelor 1967), we specify $M L_{0}$ and $S t_{0}$, which for a given $\rho_{l}$ determine the number of drops, $N_{0}$, and $D_{0}$. Because of the larger $\rho_{l}$ at the same $S t_{0}$, the MC calculations are initialized with a larger $N_{0}$ and a smaller $\left\{\left\{D_{0}\right\}\right\}$ (where $\{\{\}\}$ denotes ensemble averaging over the drops) than their SC counterpart. Since $S t$ measures the drop/flow interaction, initializing all computations with the same $S t_{0}$, specified here to give a polydisperse drop-size distribution (table 1), 


\begin{tabular}{lcccccccc}
\hline \multicolumn{1}{c}{ Case } & Fuel & $T_{0}$ & $\rho_{l}$ & $\left\{\left\{D_{0}\right\}\right\}$ & $\theta_{l, 0} / \sigma_{l, 0}$ & $\theta_{v, 0} / \sigma_{v, 0}$ & $X_{v, 0}$ & $N_{0}$ \\
SCdec375 & n-decane & 375 & 642 & $1.365 \times 10^{-4}$ & $142 / \mathrm{NA}$ & $142 / \mathrm{NA}$ & $10^{-4}$ & $580 \times 10^{3}$ \\
MCdie375 & diesel & 375 & 828 & $1.202 \times 10^{-4}$ & $185.0 / 43.0$ & $140.0 / 28.0$ & $10^{-4}$ & $670 \times 10^{3}$ \\
MCdie375x & diesel & 375 & 828 & $1.202 \times 10^{-4}$ & $185.0 / 43.0$ & $140.0 / 28.0$ & $10^{-3}$ & $670 \times 10^{3}$ \\
MCdie400 & diesel & 400 & 828 & $1.164 \times 10^{-4}$ & $185.0 / 43.0$ & $140.0 / 28.0$ & $10^{-4}$ & $690 \times 10^{3}$ \\
MCdie425 & diesel & 425 & 828 & $1.129 \times 10^{-4}$ & $185.0 / 43.0$ & $140.0 / 28.0$ & $10^{-4}$ & $720 \times 10^{3}$ \\
MCjetA375 & Jet A & 375 & 800 & $1.223 \times 10^{-4}$ & $161.0 / 29.7$ & $131.4 / 22.4$ & $10^{-4}$ & $675 \times 10^{3}$ \\
MCjetA400 & Jet A & 400 & 800 & $1.184 \times 10^{-4}$ & $161.0 / 29.7$ & $131.4 / 22.4$ & $10^{-4}$ & $695 \times 10^{3}$ \\
MCrp1375 & RP-1 & 375 & 800 & $1.223 \times 10^{-4}$ & $165.2 / 17.7$ & $153.5 / 14.8$ & $10^{-4}$ & $675 \times 10^{3}$ \\
MCrp1400 & RP-1 & 400 & 800 & $1.184 \times 10^{-4}$ & $165.2 / 17.7$ & $153.5 / 14.8$ & $10^{-4}$ & $695 \times 10^{3}$ \\
MCjp7375 & JP-7 & 375 & 800 & $1.223 \times 10^{-4}$ & $167.1 / 19.2$ & $153.7 / 15.7$ & $10^{-4}$ & $675 \times 10^{3}$ \\
MCjp7400 & JP-7 & 400 & 800 & $1.184 \times 10^{-4}$ & $167.1 / 19.2$ & $153.7 / 15.7$ & $10^{-4}$ & $695 \times 10^{3}$
\end{tabular}

TABLE 1. Initial conditions. $T_{0}$ in $\mathrm{K}, \rho_{l}$ in $\mathrm{kg} \mathrm{m}^{-3}, D_{0}$ in $\mathrm{m}$ and mean molar mass and standard deviations in $\mathrm{kg} \mathrm{kmol}^{-1}$. In all simulations, $M_{c, 0}=0.4, \delta_{\omega, 0}=6.859 \times 10^{-3} \mathrm{~m},\left\{\left\{S t_{0}\right\}\right\}=3$ and $\left\{\left\{\left(S t_{0}-\left\{\left\{S t_{0}\right\}\right\}\right)^{2}\right\}\right\}^{1 / 2}=0.5, R e_{0}=200, M L_{0}=0.2, T_{d, 0}=345 \mathrm{~K}, \gamma=86 \mathrm{~kg} \mathrm{kmol}^{-1}$ for diesel, $\gamma=41 \mathrm{~kg} \mathrm{kmol}^{-1}$ for Jet A, and $\gamma=93 \mathrm{~kg} \mathrm{kmol}^{-1}$ for RP-1 and JP-7, the grid $=200 \times 224 \times 120$, and CPU time $\simeq 792$ hours on a SGI Origin 2000 and 274 hours on a cluster with Intel Itenium2 processors.

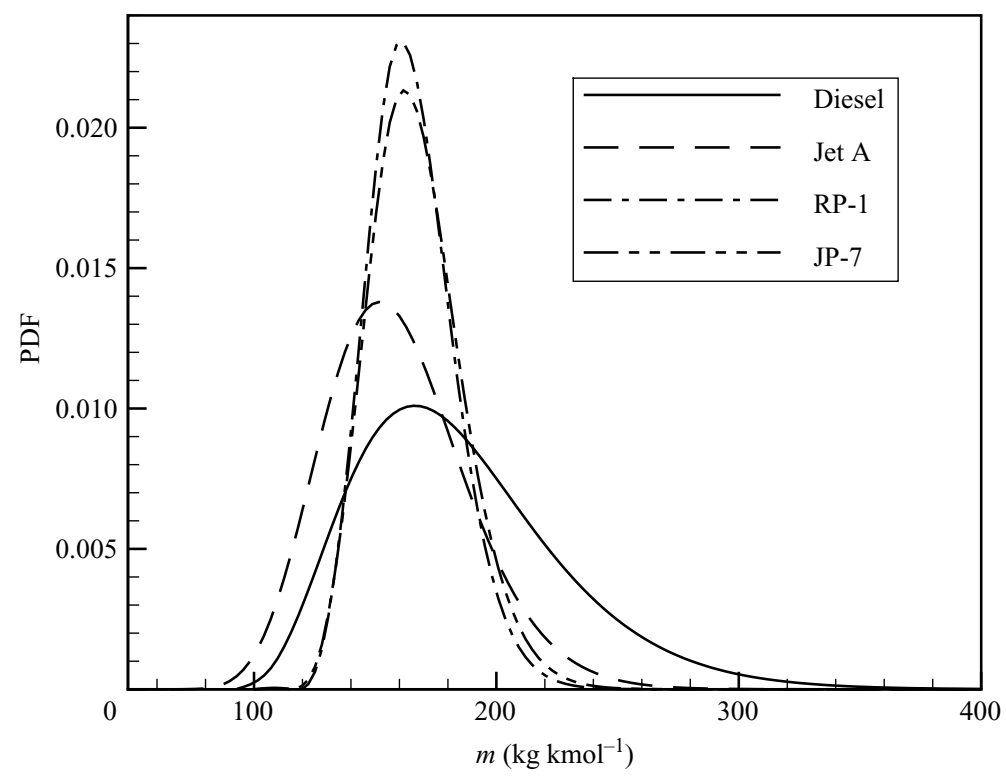

FIGURE 2. Liquid initial PDF for each fuel.

means that if differences in the flow evolution occur, they are entirely the result of the SC versus MC aspect. The MC liquids considered are diesel (Harstad et al. 2003) and three kerosenes (Jet A, RP-1 and JP-7) whose composition (Edwards \& Maurice 2001), provided as a mole fraction versus the carbon number by Edwards (2002, personal communication) was fitted in PDF form by Harstad \& Bellan (2004). All $P_{l, 0}$ are chosen to be SGPDFs as shown in figure 2 (mean and variance in table 1 ), this being an excellent representation for JP-7 (for which $\varepsilon=0$ ), a very good assumption for RP-1 (for which $\varepsilon=7.35 \times 10^{-3}$ ) and an approximation for Jet A (for which $\varepsilon=0.1357$ ) (see Harstad \& Bellan 2004). This choice means that deviations 
from an SGPDF are entirely the result of condensation on the drops. The PDF of the SC liquid (not shown) used for comparison, n-decane, would simply be a delta function at $m=142 \mathrm{~kg} \mathrm{kmol}^{-1}$; however, since a delta function is computationally intractable, the SC model of Okong'o \& Bellan (2004) is used instead.

Initially, the gas phase consists of a carrier gas (air) with a trace of vapour, $X_{v, 0}$. The trace of vapour can be chosen arbitrarily small to simulate unvitiated air, but a finite value is necessary for numerical stability purposes to initiate the calculation. The free-stream velocity $U_{0}=M_{c, 0} a_{a, 0}$ is calculated from a specified value of the convective Mach number $M_{c, 0}$ based on the carrier-gas initial speed of sound $a_{a, 0}=$ $\sqrt{R_{a} T_{0} C_{p, a} / C_{V, a}}$ at the initial uniform pressure where $R_{a}=R_{u} / m_{a}$. The specified value of the initial Reynolds number, $R e_{0}=\rho_{0} \Delta U_{0} \delta_{\omega, 0} / \mu$, where $\rho_{0}$ is the initial gas density, is used to calculate $\mu$. The thermal conductivity and diffusivity are then computed using this value of $\mu$ and (constant) specified values of $\operatorname{Pr}=S c$, computed as in Miller, Harstad \& Bellan (1998). For each MC liquid, the free-stream initial vapour composition is found from a single-drop simulation in air at the specified $T_{0}$ by choosing it to be the first-time-step surface-vapour composition; this choice means that all simulations are initiated with a uniform vapour composition between each drop surface and the drop far field represented by the gas in the lower stream.

The value of $\left(T_{0}-T_{d, 0}\right)$ is selected to ensure that the drop/flow interaction is captured over the entire layer evolution and that a substantial number of drops remains in the simulation at all times; practically, for the chosen $T_{d, 0}=345 \mathrm{~K}$, such conditions were satisfied for diesel and all kerosenes when $T_{0}=375 \mathrm{~K}, 400 \mathrm{~K}$ and $425 \mathrm{~K}$. However, n-decane simulations at $T_{0}=400 \mathrm{~K}$ led to a substantial number of drops being evaporated before the second pairing, owing to the constant value of $T_{b}=447.7 \mathrm{~K}$ which is close to $T_{0}$, and so those results were not conducive to investigating drop/flow interactions; that case was thus not included in the study. Therefore, whereas n-decane simulations could only be conducted with $\left(T_{0}-T_{d, 0}\right) / T_{0}$ of $8 \%$, for all MC liquids, additional simulations were conducted with values of $13.75 \%$ and furthermore, for diesel, as an example, also with a value of $18.82 \%$. Even for the MC liquids, higher values of $T_{0}$ were not conducive to studying drop/flow interactions because it is only in the chosen $T_{0}$ range that we could match the drop characteristic time to the relatively large flow time (i.e. $R e$ was relatively small compared to full turbulence values) in these pre-transitional simulations. All thermodynamic properties were calculated as in Harstad et al. (2003) or Miller \& Bellan (2000) and are given in Appendix A.

The boundary conditions in the $x_{1}$ - and $x_{3}$-directions are periodic, and adiabatic slip-wall conditions in the $x_{2}$-direction previously derived (Poinsot \& Lele 1992; Baum, Poinsot \& Lele 1994) were here adapted to the DGPDF CT model for MC mixtures, as described in Appendix B. Drops reaching the slip walls are assumed to stick to them and are otherwise followed using the drop equations of $\S 2.2$.

The equations were solved using an eighth-order central finite difference discretization in space and a fourth-order Runge-Kutta scheme for temporal advancement. To mitigate potential numerical instabilities for long CPU time simulations, following Kennedy \& Carpenter (1994), we used a tenth-order filtering for spatial derivatives (except in a half-filter-size band located at the lower and upper $x_{2}$ boundaries) at every time step. This filtering introduces a small amount of dissipation that serves only to stabilize the computations for long-time integrations, but since it acts only on the shortest waves that can be resolved on the grid, it does not act as a turbulence model allowing under-resolved computations (see Okong'o \& Bellan 2004). The time step was controlled by the CFL number. The grid resolution is listed in table 1 . The grid size was found by scaling approximately linearly with $R e$ (see Tennekes \& Lumley 1972) 
the previous grid of Le Clercq \& Bellan (2004) where a transitional state was achieved, manifested by a smooth velocity-fluctuation-based spectrum with no accumulation of energy at the smallest scales. The present grid size is approximately $10^{-3} \mathrm{~m}$, and considering $\left\{\left\{D_{0}\right\}\right\}$ of table 1 , the ratio of $\left\{\left\{V_{d 0}\right\}\right\}$ to the Eulerian mesh volume is approximately $10^{-3}$ and decreasing with $t$ as the drops evaporate. A fourth-order Lagrange interpolation procedure was used to obtain gas-phase variable values at drop locations. Drops whose mass decreased below $3 \%$ of the initial mass $M_{d, 0}$ were removed from the calculation; for the conditions of this study, few drops fell below $3 \%$ of $M_{d, 0}$ and mass was conserved in the system to a relative error of $10^{-3}$. A posteriori assessments of the error introduced by this drop removal criterion indicates that the error is negligible; for example, in the MCdie425 simulation, which is conducive to the highest diesel evaporation rate, the ratio $N / N_{0}$ is 0.99997 at the end of the simulation, corresponding to removing 22 drops from the initial $720 \times 10^{3}$ in the computational domain.

\subsection{Global layer evolution}

\subsubsection{Growth and dynamics}

The layer growth, measured by the momentum thickness, $\delta_{m}$, calculated as

$$
\delta_{m}=\frac{\int_{x_{2}, \min }^{x_{2}, \max }\left[\left\langle\rho u_{1}\right\rangle_{x_{2}, \max }-\left\langle\rho u_{1}\right\rangle\right]\left[\left\langle\rho u_{1}\right\rangle-\left\langle\rho u_{1}\right\rangle_{x_{2}, \min }\right] \mathrm{d} x_{2}}{\left(\left\langle\rho u_{1}\right\rangle_{x_{2}, \max }-\left\langle\rho u_{1}\right\rangle_{x_{2}, \min }\right)^{2}}
$$

where $x_{2, \max }=L_{2} / 2$ and $x_{2, \min }=-L_{2} / 2$ are the slip wall coordinates, peaks at $t^{*} \equiv$ $t \Delta U_{0} / \delta_{\omega, 0}=95$ independently of the drop composition, as shown in figure 3(a) depicting $\delta_{m} / \delta_{\omega, 0}$ for all runs having $T_{0}=375 \mathrm{~K}$ (results for $T_{0}=400 \mathrm{~K}$ are similar). Figure $3(b)$ illustrates $\delta_{m} / \delta_{\omega, 0}$ for $n$-decane and all diesel runs, and in conjunction with figure $3(a)$ leads to the conclusion that there is little layer growth sensitivity to liquid composition differences, to changes in $X_{v, 0}$ by an order of magnitude or to a variation in $\left(T_{0}-T_{d, 0}\right) / T_{0}$ of up to $18.82 \%$, although with increasing $T_{0}$ the layer becomes slightly more difficult to entrain after the first pairing. The product thickness, $\delta_{P}$

$$
\delta_{P}=\int_{0}^{L_{3}} \int_{x_{2}, \min }^{x_{2, \max }} \int_{0}^{L_{1}} \rho\left[2 \min \left(Y_{v}, Y_{a}\right)\right] \mathrm{d} x_{1} \mathrm{~d} x_{2} \mathrm{~d} x_{3},
$$

measuring the global mixing, is plotted in figures $3(c)$ and $3(d)$. In contrast to $\delta_{m} / \delta_{\omega, 0}$, $\delta_{P} / \delta_{\omega, 0}$ demonstrates substantial variability with the initial conditions and the specific liquid. For $T_{0}=375 \mathrm{~K}$, n-decane displays the best global mixing (figure $3 c$ ), this being due to its higher evaporation rate (see below), generally followed by Jet A, RP-1, JP-7 and diesel. Differences between simulations with specific liquids occur early in the layer evolution, and this high $\delta_{P}$ sensitivity may therefore constitute a good experimental diagnostic for comparing simulations with liquids of different volatilities. The early higher $\delta_{P}$ for Jet $\mathrm{A}$ compared to n-decane results from the initially higher evaporation rate, as lower-molar-mass species than n-decane, which are more volatile, egress the Jet A drops. The final ordering of $\delta_{P}$ values is entirely correlated with the anticipated liquid saturation curve; this saturation curve is fixed for n-decane but continuously evolves with time for MC liquids due to the change in composition. Among the diesel simulations, $\delta_{P}$ increases with increasing $X_{v, 0}$ because despite the reduced drop evaporation rate (see below), the larger $Y_{v, 0}$ contributes to the larger vapour mass in the domain (not shown). Figure $3(d)$ shows that $\delta_{P} / \delta_{\omega, 0}$ 

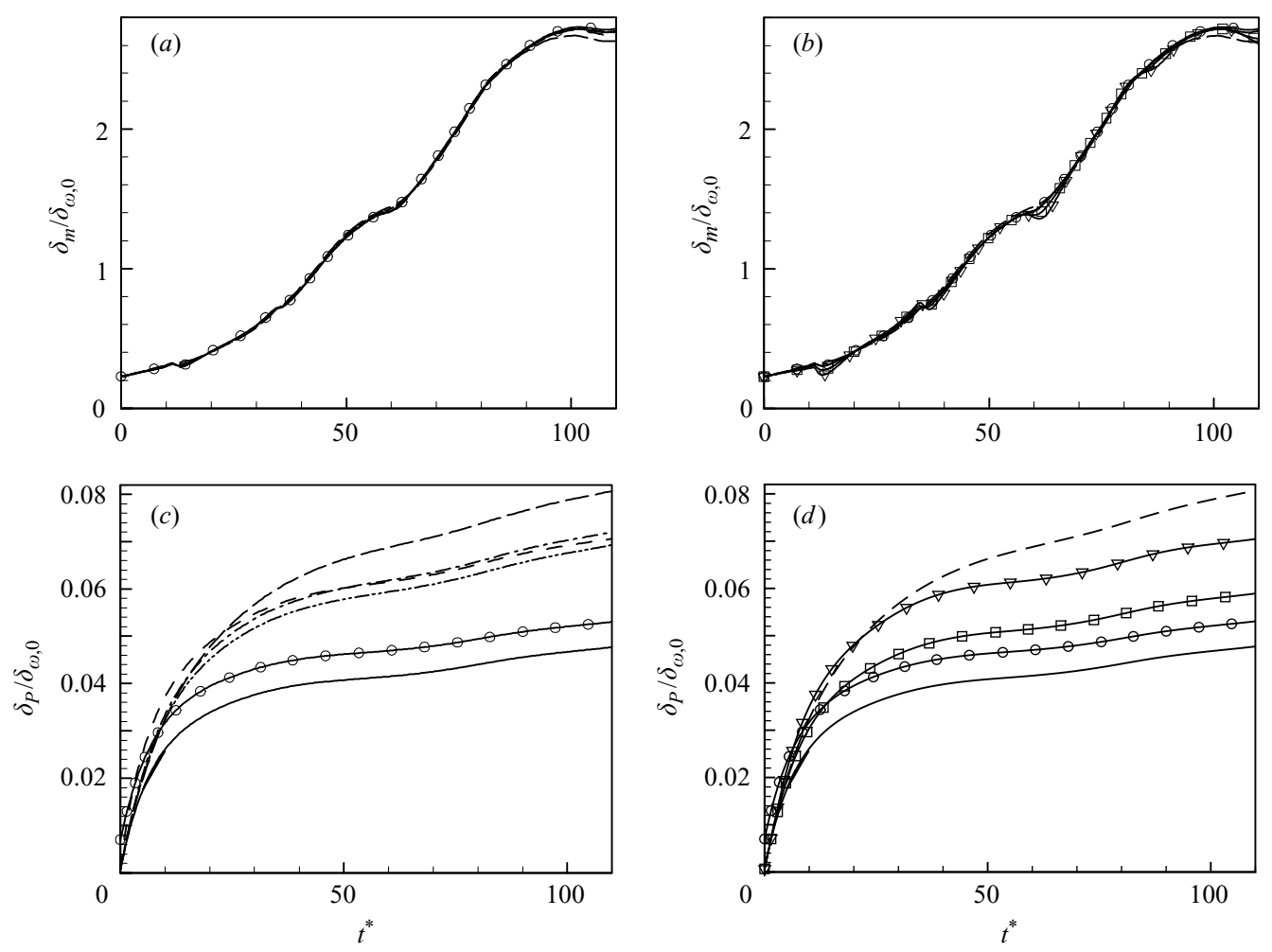

FIGURE 3. Timewise $\left(t^{*} \equiv t \Delta U_{0} / \delta_{\omega, 0}\right)$ evolution of global quantities: the momentum thickness $(a, b)$ and product thickness $(c, d)$. In $(a)$ and $(c)$ all simulations have $T_{0}=375 \mathrm{~K}$. In $(b)$ and $(d)$ are illustrated all diesel-fuel simulations and n-decane, for comparison. - _ - SCdec 375; $\longrightarrow$, MCdie 375; ———-, MCdie375x; - - - MCdie400; — -, MCdie425; - - - -, MCjetA375;-.---, MCrp1375; -..-..-, MCjp7375.

increases with $T_{0}$, which results from the larger evaporation rate. It is also apparent that $\delta_{P} / \delta_{\omega, 0}$ for n-decane considerably exceeds that of diesel at the same $T_{0}$, this being due to the diesel much smaller volatility. This indicates that SC liquids are poor substitutes for MC liquids for simulating phenomena associated with phase change.

Rotational characteristics of the layer are depicted in figure 4: the positive spanwise vorticity, $\left\langle\left\langle\omega_{3}^{+}\right\rangle\right\rangle$in figures $4(a), 4(c), 4(e)$, and the enstrophy, $\left\langle\left\langle\omega_{i} \omega_{i}\right\rangle\right\rangle$ in figures $4(b)$, $4(d), 4(f)$, where $\langle\langle\rangle\rangle$ denotes averaging over all grid points. Initially null, $\omega_{3}^{+}$is a measure of the small-scale activity; $\omega_{i} \omega_{i}$ is related to stretching and tilting which is an important mechanism for turbulence production (see Chorin 1991). For all simulations, $\left\langle\left\langle\omega_{3}^{+}\right\rangle\right\rangle \delta_{\omega, 0} / \Delta U_{0}$ grows to an initial peak at around the time of the first pairing, exhibits thereafter a smaller rate of growth, this being the effect of forcing, and then grows again to a higher second peak, after which it declines. The time of the second peak approximately corresponds to the time of the second pairing. After the second pairing, n-decane exhibits a decline, unlike the MC liquids (figure $4 a$ ). Diesel displays a slightly reduced peak compared to all other liquids and its value seems insensitive to $X_{v, 0}$ in the studied range (figure $4 a$ ). Since diesel has similar $\rho_{l}$ to the kerosenes and the simulations are initiated with similar $N_{0}$ and $\left\{\left\{D_{0}\right\}\right\}$, it appears that there may be composition effects at play, which persist at the larger $T_{0}=400 \mathrm{~K}$ 

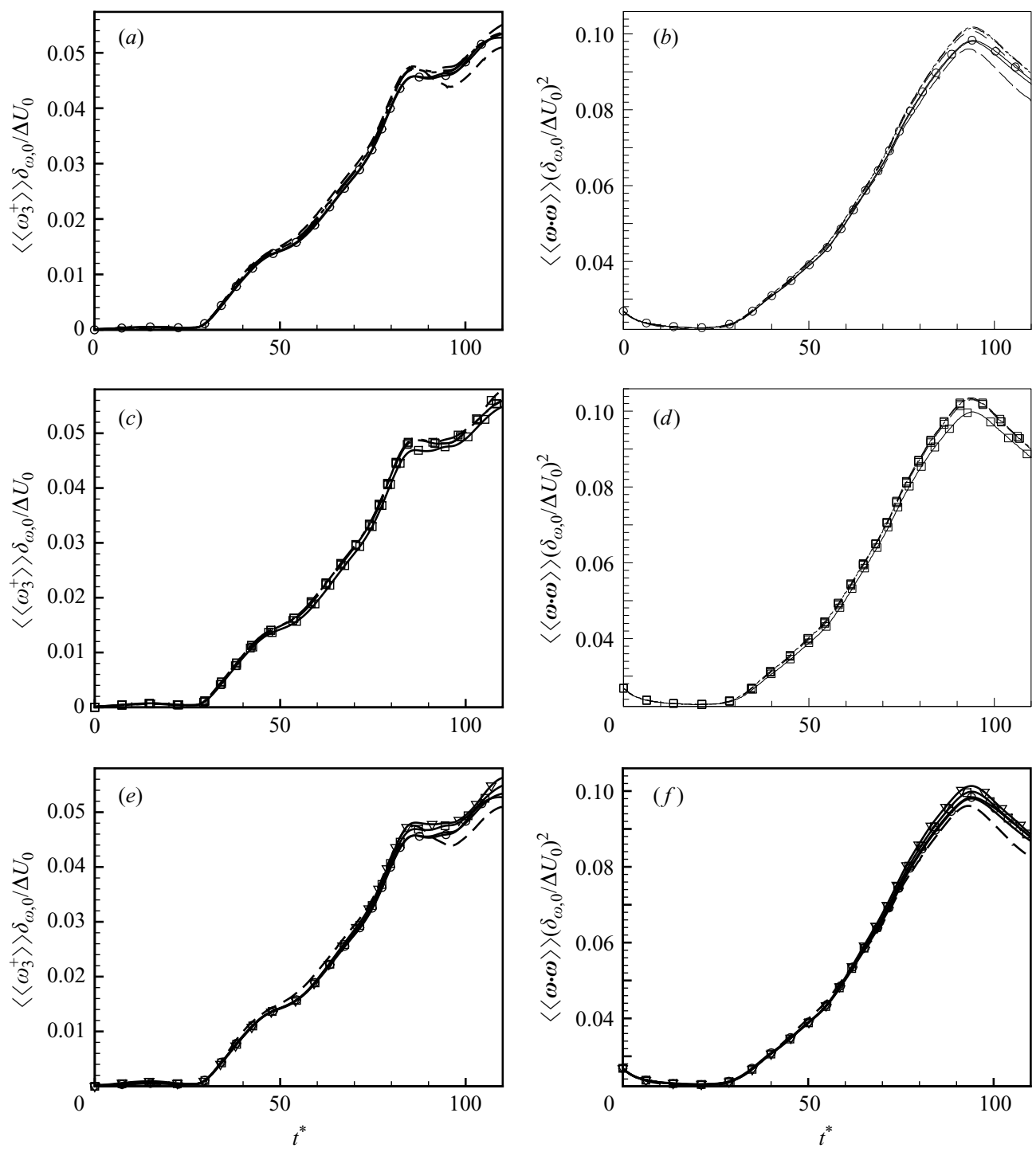

FIgURE 4. Timewise evolution of the Eulerian average positive spanwise vorticity $(a, c, e)$ and the enstrophy $(b, d, f)$, for $(a)$ and $(b)$ all simulations having an initial temperature of $375 \mathrm{~K},(c)$ and $(d)$ all simulations having an initial temperature of $400 \mathrm{~K}$, and $(e)$ and $(f)$ all

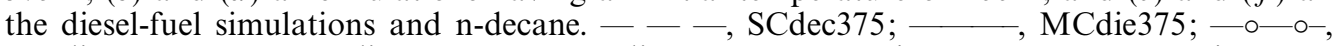
MCdie375x; - - -, MCdie400;- - -, MCdie425;----, MCjetA375;- $\square-\square-$, MCjetA400;

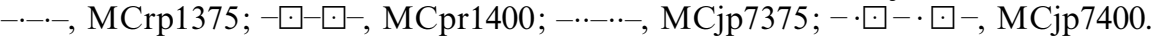

(figure $4 c$ ). With increasing $T_{0}$, the magnitude of $\left\langle\left\langle\omega_{3}^{+}\right\rangle\right\rangle$increases at the second peak (figure $4 e$ ), and at the higher $T_{0}$, the second peak has a longer lifetime and leads to higher growth thereafter. Regarding $\left\langle\left\langle\omega_{i} \omega_{i}\right\rangle\right\rangle\left(\delta_{\omega, 0} / \Delta U_{0}\right)^{2}$, its peak, achieved after the second pairing, is smaller for n-decane than for all MC liquids (figure $4 b)$ and the differentiation between diesel and kerosenes is stronger than that seen for $\left\langle\left\langle\omega_{3}^{+}\right\rangle\right\rangle$, a result that is invariant with $T_{0}$ (figure $4 d$ ) and which confirms the liquid-specific enstrophy evolution (figures $4 d$ and $4 f$ ). 

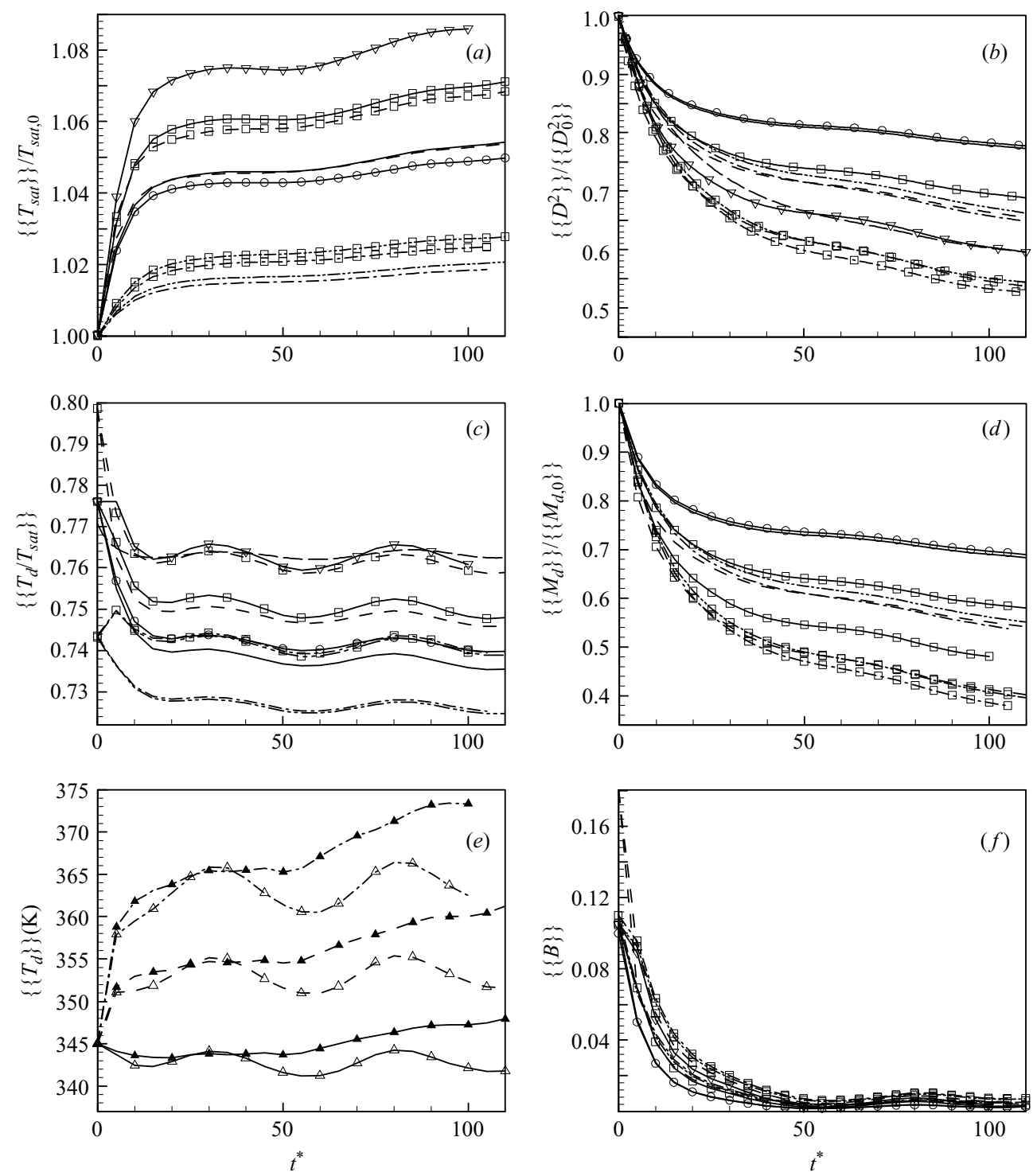

FIGURE 5. Timewise evolution of drop-ensemble averaged quantities: the normalized saturation temperature $(a)$, the normalized drop diameter square $(b)$, the ratio of the drop temperature to the saturation temperature $(c)$, the normalized drop mass $(d)$, the drop temperature in the lower stream (ls) and in the mixing layer $(\mathrm{ml})(e)$, and the transfer number $(f)$. - SCdec375; — , MCdie 375; ———-, MCdie 375x; - - - MCdie400; — - - MCdie425; ---- , MCjetA375; - $\square-\square-$, MCjetA400; -.--, MCrp1375; - $-\square-$, MCpr1400; -..-..-, MCjp7375;-·- $\cdot \square-$, MCjp7400. Legend for $(e)-\Delta-, 3751 \mathrm{~s} ;-\boldsymbol{\Delta}-, 375 \mathrm{ml} ;--\Delta--, 4001 \mathrm{~s}$; $--\boldsymbol{\Lambda}_{-}-, 400 \mathrm{ml} ;-\cdot-\Delta_{---}, 4251 \mathrm{~s} ;-\cdot-\mathbf{\Delta}_{---,}, 425 \mathrm{ml}$.

\subsubsection{Drop characteristics}

Shown in figure 5 are Lagrangian ensemble averages portraying the evolution of the drop characteristics. For n-decane, $T_{\text {sat }}$ is constant, $\left\{\left\{T_{\text {sat }}\right\}\right\} / T_{\text {sat }, 0}=1$, and is thus not plotted in figure 5(a). For all MC liquids, $\left\{\left\{T_{\text {sat }}\right\}\right\} / T_{\text {sat }, 0}$ increases rapidly during rollup because the most volatile species leave the drops, and then gradually becomes 
asymptotic, as drop composition changes become smaller with time (see discussion of figure 6). The least increase, $\sim 1.5 \%$, is observed for RP-1 and JP-7 at $T_{0}=375 \mathrm{~K}$; the larger $T_{0}=400 \mathrm{~K}$ only elevates $\left\{\left\{T_{\text {sat }}\right\}\right\} / T_{\text {sat }, 0}$ to slightly more than $2 \%$. Jet A, being more volatile than the other two kerosenes, experiences a larger change in $\left\{\left\{T_{\text {sat }}\right\}\right\}$ / $T_{\text {sat }, 0}$, with about $4.5 \%$ and $6 \%$ eventual increase at $T_{0}=375 \mathrm{~K}$ and $400 \mathrm{~K}$, respectively. The diesel profile coincides with that of Jet A at $T_{0}=375 \mathrm{~K}$, which is happenstance resulting from the ensemble averaging; at $T_{0}=400 \mathrm{~K}$, a slightly larger $\left\{\left\{T_{\text {sat }}\right\}\right\}$ / $T_{\text {sat }, 0}$ is obtained for diesel than at $T_{0}=375 \mathrm{~K}$, and the maximum value is about $6.5 \%$. The distinct behaviour of each liquid is noticeable. With increasing $T_{0}$, there is a correspondingly larger initial rate of increase in $\left\{\left\{T_{s a t}\right\}\right\} / T_{s a t, 0}$ as the volatile species evaporate faster, and the ultimate value reached is larger, being almost $9 \%$ for $T_{0}=425 \mathrm{~K}$. With increasing $X_{v, 0}$, evaporation is slower, which slightly decreases $\left\{\left\{T_{\text {sat }}\right\}\right\} / T_{\text {sat }, 0}$. Although the percentage changes in $\left\{\left\{T_{\text {sat }}\right\}\right\} / T_{\text {sat }, 0}$ are small, for individual drops, particularly in the mixing layer, they can be much larger, which affects the local composition.

Examination of $\left\{\left\{D^{2}\right\}\right\} /\left\{\left\{D_{0}^{2}\right\}\right\}$ in figure 5(b) shows that among all liquids, at fixed $T_{0}$, the $n$-decane drops evaporate fastest, reaching about $60 \%$ of $\left\{\left\{D_{0}^{2}\right\}\right\}$, which corresponds to about $46 \%$ of the initial mass as shown in figure $5(d)$ depicting $\left\{\left\{M_{d}\right\}\right\} /$ $\left\{\left\{M_{d, 0}\right\}\right\}$. The diesel drops evaporate slowest, reaching about $71 \%$ of the initial mass, and the three kerosenes evaporate at approximately the same rate, reaching $52 \%-$ $55 \%$ of the initial mass. As expected, with increasing $T_{0}$, the drops evaporate faster and for larger $X_{v, 0}$ the drops evaporate slower; however, the small differences among values of $\left\{\left\{T_{\text {sat }}\right\}\right\} / T_{\text {sat }, 0}$ displayed in figure 5(a) translate to much larger differences for $\left\{\left\{D^{2}\right\}\right\} /\left\{\left\{D_{0}^{2}\right\}\right\}$. Moreover, these $\left\{\left\{D^{2}\right\}\right\} /\left\{\left\{D_{0}^{2}\right\}\right\}$ differences are even larger if only the drops in the layer are considered (Le Clercq \& Bellan 2004), since the more numerous drops in the lower stream eventually saturate (Le Clercq \& Bellan 2004), which biases the ensemble average value, while the mixing layer drop size continuously decreases. The wide range of residual mass attained by the drops, from $38 \%$ to $72 \%$, is evident.

Presented in figure $5(c)$ is $\left\{\left\{T_{d} / T_{\text {sat }}\right\}\right\}$ versus $t^{*}$. In all cases, a very mild $\left\{\left\{T_{d} / T_{\text {sat }}\right\}\right\}$ undulatory behaviour is observed, traced by Le Clercq \& Bellan (2004) to $\left\{\left\{T_{d}\right\}\right\}$ which manifests evaporative cooling and heating cycles. Ensemble averages conditioned on drop location (mixing layer for $x_{2} / \delta_{\omega, 0} \geqslant-7$, subscript $m l$; complement is the lower stream, subscript $l s)$ displayed in figure $5(e)$ show this behaviour to be biased by the larger number of drops in $x_{2} / \delta_{\omega, 0}<-7$, as the mixing layer $\left\{\left\{T_{d}\right\}\right\}$ continuously increases after the first pairing. For n-decane, the variation of $\left\{\left\{T_{d} / T_{\text {sat }}\right\}\right\}$ (not shown) duplicates the $\left\{\left\{T_{d}\right\}\right\}$ behaviour owing to $T_{\text {sat }}$ being constant. For the MC liquids, the behaviour is due to either the counteracting or the concerting effect of $T_{d}$ and $T_{\text {sat }}$. As the volatile species preferentially leave the drops, $\left\{\left\{T_{s a t}\right\}\right\}$ increases concomitantly with $\left\{\left\{\theta_{l}\right\}\right\} / \theta_{l, 0}$ (shown below). At $T_{0}=375 \mathrm{~K}$, the initial reduction of $\left\{\left\{T_{d} / T_{\text {sat }}\right\}\right\}$ combines the decrease in $T_{d}$ with the increase in $T_{\text {sat }}$. At $T_{0}>375 \mathrm{~K}$, two initial behaviours are seen: for diesel and Jet $\mathrm{A},\left\{\left\{T_{d} / T_{\text {sat }}\right\}\right\}$ initially diminishes, meaning that the increase in $T_{\text {sat }}$ due to the evaporation of lower- $m$ species is larger than the increase in $T_{d}$ due to drop heating whereas for RP-1 and JP-7, $\left\{\left\{T_{d} / T_{\text {sat }}\right\}\right\}$ initially augments, meaning the opposite. The peaks and troughs in $\left\{\left\{T_{d} / T_{\text {sat }}\right\}\right\}$ emulate those of $\left\{\left\{T_{d}\right\}\right\}$ indicating that away from the initial condition the global $T_{d}$ variation is larger than that of $T_{\text {sat }}$.

Considering the range of $T_{d}$ in figure 5(e) one can now evaluate the assumption of negligible liquid thermal expansion, $\alpha_{V_{d}}$, effect on the change in drop size. Because

$$
\alpha_{V_{d}} \equiv\left[\partial\left(\ln V_{d}\right) / \partial T\right]_{p, X}, \quad \text { then } V_{d}\left(T_{d}\right)=V_{d}\left(T_{d 0}\right) \exp \left(\int_{T_{d 0}}^{T_{d}} \alpha_{V_{d}} \mathrm{~d} T\right) \text {. }
$$


Known values of $\alpha_{V_{d}}$ for several hydrocarbons are: (i) for toluene $1.05 \times 10^{-3} \mathrm{~K}^{-1}$ at $298 \mathrm{~K}$ and $1.13 \times 10^{-3} \mathrm{~K}^{-1}$ at $323 \mathrm{~K}$, and (ii) for octane $1.16 \times 10^{-3} \mathrm{~K}^{-1}$ at $298 \mathrm{~K}$ and $1.23 \times 10^{-3} \mathrm{~K}^{-1}$ at $318 \mathrm{~K}$; higher- $m$ paraffin hydrocarbons have somewhat smaller $\alpha_{V_{d}}$ values. Assuming an average $\alpha_{V_{d}}=1.00 \times 10^{-3} \mathrm{~K}^{-1}$ for diesel, and calculating $V_{d}\left(T_{d}\right) /$ $V_{d}\left(T_{d 0}\right)$ for $T_{d}=375 \mathrm{~K}$ (the $T_{d}$ upper-bound value in figure $5 e$ ), one finds $V_{d}\left(T_{d}\right) /$ $V_{d}\left(T_{d 0}\right) \simeq 1.03$, corresponding to an average $D / D_{0} \simeq 1.01$. This a posteriori evaluation shows that thermal expansion negligibly affects the drop size in the present simulations.

The ensemble-average Spalding number is presented in figure $5(f)$. The initial value of $\{\{B\}\}$ for Jet $\mathrm{A}$ is larger than for all other liquids because of its higher volatility. For all simulations, $\{\{B\}\}$ is small compared to unity and continuously and drastically decreases, verifying a posteriori the assumption of drop-interior uniform conditions (Makino \& Law 1988). The largest rate of decline occurs for the diesel simulation with $X_{v, 0}=10^{-3}$, consistent with the decreased evaporation. When calculating the ensemble average of $B$ using only the drops in the lower stream, and for all simulations, small negative values are reached after the first and second pairings (not shown), indicating that at those times the liquid composition change in the lower stream is dominated by condensation. Except for Jet A, the magnitude of the negative values increases with increasing $T_{0}$ which is attributed to the increasing evaporation rate that brings lighter species into the gas phase that then proceed to condense on the drops. Companion plots of $\left\{\left\{D^{2}\right\}\right\} /\left\{\left\{D_{0}^{2}\right\}\right\}$ ensemble averaged over the lower stream (not shown), show an asymptotic value eventually reached, which supports this interpretation.

The evolution of the liquid composition is displayed in figure 6 in terms of $\left\{\left\{\theta_{l}\right\}\right\} / \theta_{l, 0}$ and $\left\{\left\{\sigma_{l}\right\}\right\} / \sigma_{l, 0}$. The initial $\left\{\left\{\theta_{l}\right\}\right\} / \theta_{l, 0}$ surge is accompanied by a drastic reduction in $\left\{\left\{\sigma_{l}\right\}\right\} / \sigma_{l, 0}$ as the volatiles evaporate, thus reducing the number of species in the drop, and eventually $\left\{\left\{\sigma_{l}\right\}\right\} / \sigma_{l, 0}$ reaches a minimum. This minimum, beyond which $\left\{\left\{\sigma_{l}\right\}\right\} / \sigma_{l, 0}$ continuously increases, coincides with a tapering off in the augmentation of $\left\{\left\{\theta_{l}\right\}\right\} / \theta_{l, 0}$. Thereafter, both evaporation and condensation occur: condensation increases $\left\{\left\{\sigma_{l}\right\}\right\} / \sigma_{l, 0}$ whereas evaporation of the lighter species increases $\left\{\left\{\theta_{l}\right\}\right\} / \theta_{l, 0}$. Most changes in $\left\{\left\{\theta_{l}\right\}\right\} / \theta_{l, 0}$ occur for diesel and Jet $\mathrm{A}$, and are about $10 \%$ at $T_{0}=375 \mathrm{~K}$ and $13 \%$ at $T_{0}=400 \mathrm{~K}$, while the corresponding values for RP- 1 and JP-7 are lower than $4 \%$ and $6 \%$, respectively (figures $6 a$ and $6 c$ ). For the diesel simulation at $T_{0}=425 \mathrm{~K}$, in excess of $17 \%$ change in $\left\{\left\{\theta_{l}\right\}\right\} / \theta_{l, 0}$ is observed (figure $6 e$ ). These variations in $\left\{\left\{\theta_{l}\right\}\right\} / \theta_{l, 0}$ and $\left\{\left\{\sigma_{l}\right\}\right\} / \sigma_{l, 0}$ are ensemble averages, and therefore for some drops, individual variations are larger. Minimal sensitivity is exhibited by $\left\{\left\{\theta_{l}\right\}\right\} / \theta_{l, 0}$ to an order of magnitude increase in $X_{v, 0}$ (figures $6 a$ and $6 e$ ) because, as shown by Harstad et al. (2003) for a single drop, it is the composition of the vapour rather than the value of the far-field vapour mole fraction (in this regime) that affects $\left\{\left\{\theta_{l}\right\}\right\} / \theta_{l, 0}$; the small decrease in $\left\{\left\{\theta_{l}\right\}\right\} / \theta_{l, 0}$ occurring with increasing $X_{v, 0}$ results from the reduced evaporation rate which thus promotes a correspondingly smaller change in composition. Of note is that $\left\{\left\{\sigma_{l}\right\}\right\} / \sigma_{l, 0}$ exhibits more sensitivity to all parameters than $\left\{\left\{\theta_{l}\right\}\right\} / \theta_{l, 0}$. At both $T_{0}=375 \mathrm{~K}$ and $400 \mathrm{~K}$ (figures $6 b$ and $6 d$ ), Jet $\mathrm{A}$ has the largest initial decline in $\left\{\left\{\sigma_{l}\right\}\right\} / \sigma_{l, 0}$ (in excess of $7 \%$ and $8 \%$, respectively) as the stronger evaporation depletes more of the volatiles compared to the other liquids. With condensation, $\left\{\left\{\sigma_{l}\right\}\right\} / \sigma_{l, 0}$ is augmented again, and this recovery is stronger with increasing $T_{0}$ owing to the larger range of volatile species that may condense. Diesel displays less $\left\{\left\{\sigma_{l}\right\}\right\} / \sigma_{l, 0}$ variation than Jet A during the layer evolution and its initial decline as well as the recovery are smaller. Both RP-1 and JP-7 show the least initial reduction in $\left\{\left\{\sigma_{l}\right\}\right\} / \sigma_{l, 0}$ due to their narrower composition PDF, and the continuous increase past unity indicates that the initial SGPDF peak has been substantially 

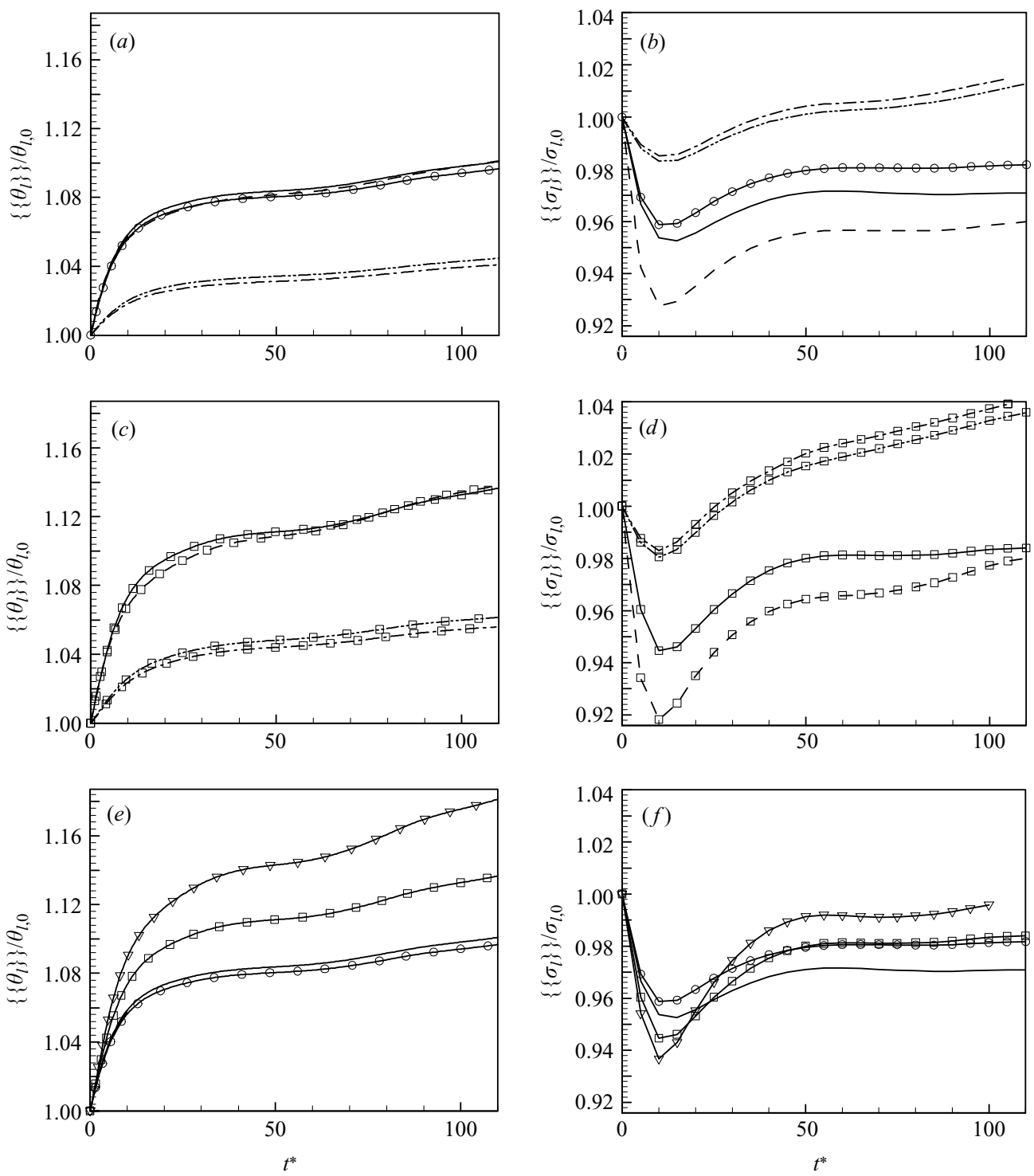

FiguRE 6. Timewise evolution of drop-ensemble-averaged quantities: the average liquid mean molar mass $(a, c, e)$ and standard deviation $(b, d, f)$. ——, MCdie 375; — ○ ०-, MCdie 375x; $-\square-$, MCdie400; - $\nabla-$, MCdie425; - - - , MCjetA375; - $\square-\square-$, MCjetA400; -.---,

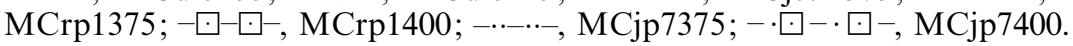

reduced to permit a non-negligible contribution to the PDF by increasingly lower$m$ species. The percentage augmentation of $\left\{\left\{\sigma_{l}\right\}\right\} / \sigma_{l, 0}$ past unity increases with $T_{0}$ because the promoted evaporation results in increasingly less volatile species leaving the drops. For diesel, the increasing $T_{0}$ leads to a greater effect in the initial reduction of $\left\{\left\{\sigma_{l}\right\}\right\} / \sigma_{l, 0}$ and to a larger recovery (figure $6 f$ ), indicating the strong temperature effect on evaporation and condensation, particularly for the lower- $m$ species, and thus the substantial $T_{0}$ influence on the liquid composition.

The conclusion is that liquid specificity is important in determining both the drop physical characteristics and the liquid composition, and that $T_{0}$ has a large impact 

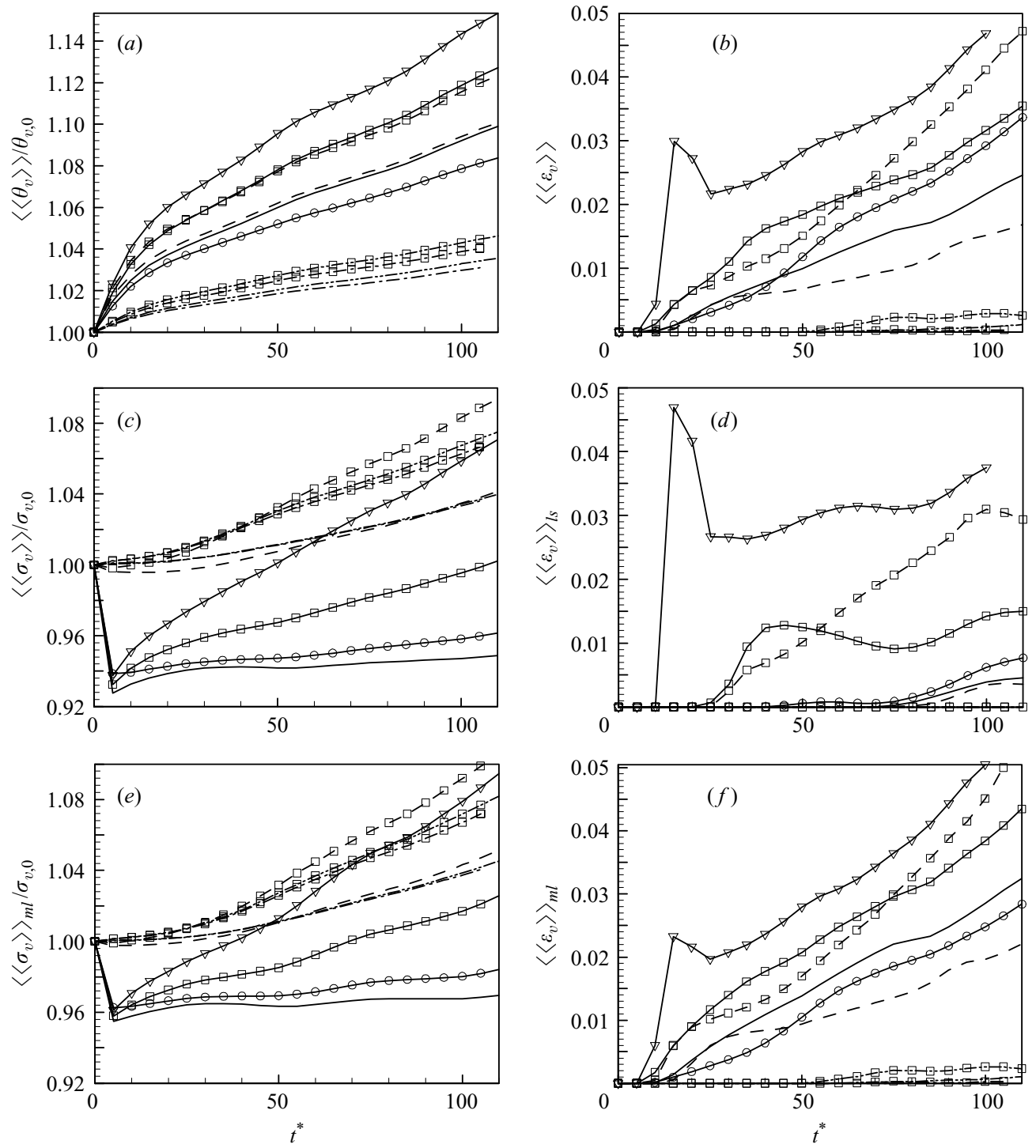

FigurE 7. Timewise evolution of the volume-average vapour mean molar mass $(a)$, the volume average parameter $\varepsilon(b)$, the normalized volume average standard deviation $(c)$, the volume average parameter $\varepsilon$ in the lower stream $(d)$, the normalized volume average standard deviation in the mixing layer $(e)$, and the volume average parameter $\varepsilon$ in the mixing layer $(f)$. MCdie375; ———-, MCdie375x; - $\square-$, MCdie400; - - - MCdie425; - - -, MCjetA375;

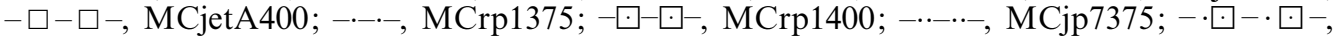
MCjp7400.

on the liquid composition evolution. The relatively small variations observed here correspond to the small $\left(T_{0}-T_{d, 0}\right) / T_{0}$ range investigated, and it is predictable that larger variations will occur at higher $\left(T_{0}-T_{d, 0}\right) / T_{0}$.

\subsubsection{Vapour composition characteristics}

Figure 7 shows the volumetric evolution of the vapour composition resulting from drop evaporation. The results were obtained by post-processing the database, which 
explains some sharp profiles produced by information available only every 5 units of $t^{*}$. In all simulations, $\left\langle\left\langle\theta_{v}\right\rangle\right\rangle / \theta_{v, 0}$ (figure $7 a$ ) increases and duplicates the $\left\{\left\{\theta_{l}\right\}\right\} / \theta_{l, 0}$ results in terms of relative variation among liquids. The RP-1 and JP-7 composition increase slowest, with little impact of $T_{0}$ in the range examined; diesel and Jet A have similar volumetric variation and a much higher response to $T_{0}$ than RP-1 and JP-7; and an increase in $X_{v, 0}$ reduces $\left\langle\left\langle\theta_{v}\right\rangle\right\rangle / \theta_{v, 0}$. However, in contrast to $\left\{\left\{\theta_{l}\right\}\right\} / \theta_{l, 0}$, the variation of which subsided with time, $\left\langle\left\langle\theta_{v}\right\rangle\right\rangle / \theta_{v, 0}$ continuously increases at an almost constant rate, reaching as much as $14 \%$ for diesel at $T_{0}=425 \mathrm{~K}$. This continuous augmentation occurs due to the evaporation of increasingly larger- $m$ fuel species, since the lighter species have been released early during the drop lifetime. A large differentiation is observed in figure $7(c)$ between the evolution of $\left\langle\left\langle\sigma_{v}\right\rangle\right\rangle / \sigma_{v, 0}$ for diesel and for the kerosenes. A sharp initial decline from the free-stream values occurs for diesel, which indicates initial condensation of the lighter vapour species onto the drops; this condensation is concurrent with evaporation of the lighter species from the drops (figure 6) leading to net evaporation (figure $5 b$ ). Following the initial vapour condensation, $\left\langle\left\langle\sigma_{v}\right\rangle\right\rangle / \sigma_{v, 0}$ increases, indicating a more elevated heterogeneous composition of the vapour with time, with substantial $T_{0}$ and little $X_{v, 0}$ impact. In contrast to diesel, Jet A experiences negligible initial condensation; RP-1 and JP-7, the $\left\langle\left\langle\sigma_{v}\right\rangle\right\rangle / \sigma_{v, 0}$ evolutions of which coincide, do not display initial condensation at all. For all kerosenes, $T_{0}$ has a smaller impact on the vapour composition compared to diesel. To inquire into the specific fate of the mixing layer vapour, in figure 7(e) is plotted $\left\langle\left\langle\sigma_{v}\right\rangle\right\rangle_{m l} / \sigma_{v, 0}$ which shows a smaller initial decline than $\left\langle\left\langle\sigma_{v}\right\rangle\right\rangle / \sigma_{v, 0}$ and which displays a greater augmentation at the end of the simulation. These observations indicate that most condensation occurs in the lower stream, and that the vapour in the mixing layer is more heterogeneous in composition than that in the lower stream.

The departure of the vapour composition from the SGPDF is documented in figures $7(b), 7(d)$ and $7(e)$ where $\left\langle\left\langle\varepsilon_{v}\right\rangle\right\rangle,\left\langle\left\langle\varepsilon_{v}\right\rangle\right\rangle_{l s}$ and $\left\langle\left\langle\varepsilon_{v}\right\rangle\right\rangle_{m l}$ are plotted, respectively. The evolution of $\varepsilon_{v}$ from its initial null value documents condensation of small- $m$ species onto the drops, producing a liquid DGPDF, which then gives rise to a gas DGPDF, since as explained by Cotterman et al. (1985), the PDFs in the two phases must have the same mathematical form. Except at the higher $T_{0}$, RP-1 and JP-7 maintain a SGPDF during the entire layer evolution. At the larger $T_{0}$, the largest departures from the SGPDF occur in the mixing layer where there is concurrent condensation and evaporation, as the lower stream becomes eventually saturated (see Le Clercq \& Bellan 2004). Most of the activity leading from a SGPDF to a DGPDF for Jet A also occurs in the mixing layer, and although larger than for the other kerosenes, it is modest at $T_{0}=375 \mathrm{~K}$ but increases with $T_{0}$, reaching as much as $5 \%$ at $T_{0}=400 \mathrm{~K}$; because these results are volumetric averages, at some spatial locations, $\varepsilon_{v}$ has larger values. Diesel experiences the largest departures from the SGPDF, and these increase very strongly with $T_{0}$. Volumetrically over the entire domain, the SGPDF evolves into a DGPDF as soon as the layer rolls up, although except for $T_{0}=425 \mathrm{~K}$, most of the activity is in the mixing layer where the drops are moved by the flow and exposed to vapour of different composition. The initial surge in $\left\langle\left\langle\varepsilon_{v}\right\rangle\right\rangle$ at $T_{0}=425 \mathrm{~K}$ is consistent with the condensation indicated by the sharp increase in $\left\langle\left\langle\sigma_{v}\right\rangle\right\rangle / \sigma_{v, 0}$ past the initial transient; the following decline in $\left\langle\left\langle\varepsilon_{v}\right\rangle\right\rangle$ indicates evaporation of the lighter species, and the ultimate increase agrees with condensation being concomitant with evaporation. The lower stream experiences most initial evolution towards a DGPDF because it is more prone to condensation due to the preponderance of the lighter species; it is also in the lower stream that most tendency to return towards a SGPDF occurs due to the release of the lower- $m$ species through evaporation. Following the 

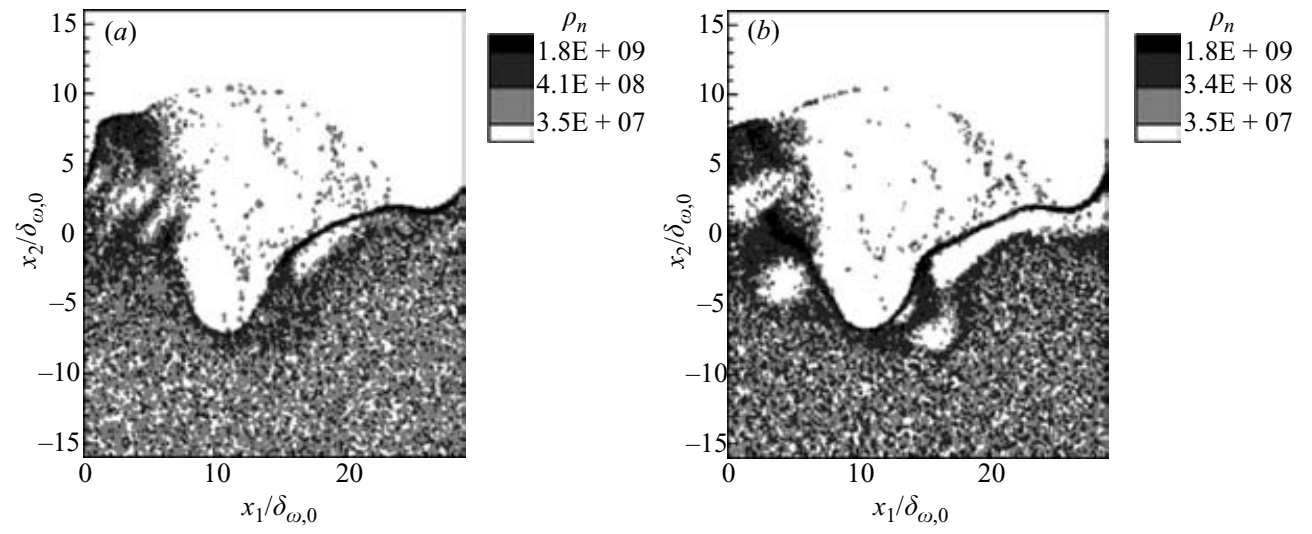

FIGURE 8. Contour plots of the drop number density in the between-the-braid plane $x_{3} / \delta_{\omega, 0}=8.75$ at $t^{*}=95$. $\operatorname{SCdec} 375(a)$, and MCdie375 $(b)$.

initial transient, the lower-stream vapour composition restarts its evolution towards a DGPDF. For diesel at $T_{0}=375 \mathrm{~K}$ and $400 \mathrm{~K}$, these stages occur later in the layer evolution and they are more subdued. Although in all simulations the $\varepsilon_{v}$ values are small, it has been shown by Harstad et al. (2003) that this small parameter embodies the ability of the model to portray the physics.

To develop an understanding of the state of the flow resulting from the liquidspecific drop/flow interaction, visualizations and homogeneous $\left(x_{1}, x_{3}\right)$-plane averages at $t^{*}=95$, are examined next.

\subsection{Flow visualizations}

\subsubsection{Drop field}

Illustrated in figures 8 and 9 is the drop number density, $\rho_{n}$, calculated as an Eulerian field from the Lagrangian distribution

$$
\rho_{n}=\sum_{q=1}^{N_{d}} \frac{w_{q}}{\Delta V_{q}} .
$$

The plots depict the between-the-braid plane $x_{3} / \delta_{\omega, 0}=8.75$ distribution for several simulations at $t^{*}=95$. In all cases, a complex drop organization is observed, with relatively void regions corresponding to locations of high vorticity (see below); these voids are profiled by high- $\rho_{n}$ regions that correspond to high-strain locations (Squires \& Eaton 1991). In all simulations, a thin braid of large $\rho_{n}$ is apparent, extending from the lower stream into the upper stream and marks the edge of the ultimate vortex.

Comparing in figure 8 the n-decane and diesel drop organization at the same $T_{0}$, it is apparent that $\rho_{n}$ is smaller and that the drop organization is less complex for n-decane. The smaller $\rho_{n}$ is the combined result of the smaller $N_{0}$ and a larger number of evaporated drops being removed from the computation (as they reach the less than $3 \%$ residual mass criterion), despite their initially larger $\left\{\left\{D_{0}\right\}\right\}$. The reduced structure observed for the n-decane drop field with respect to that for diesel occurs because of the related smaller $\{\{D\}\}$ (see figure $5 b$ ) and smaller $\rho_{n}$ resulting from drop attrition, each of which reduces the interaction between drops and flow.

The structural complexity of the drop organization is insensitive to $T_{0}$ (figures $9 a, 9 c$ and $9 e$ ), which is the consequence of two counteracting effects. The larger $N_{0}$ at 

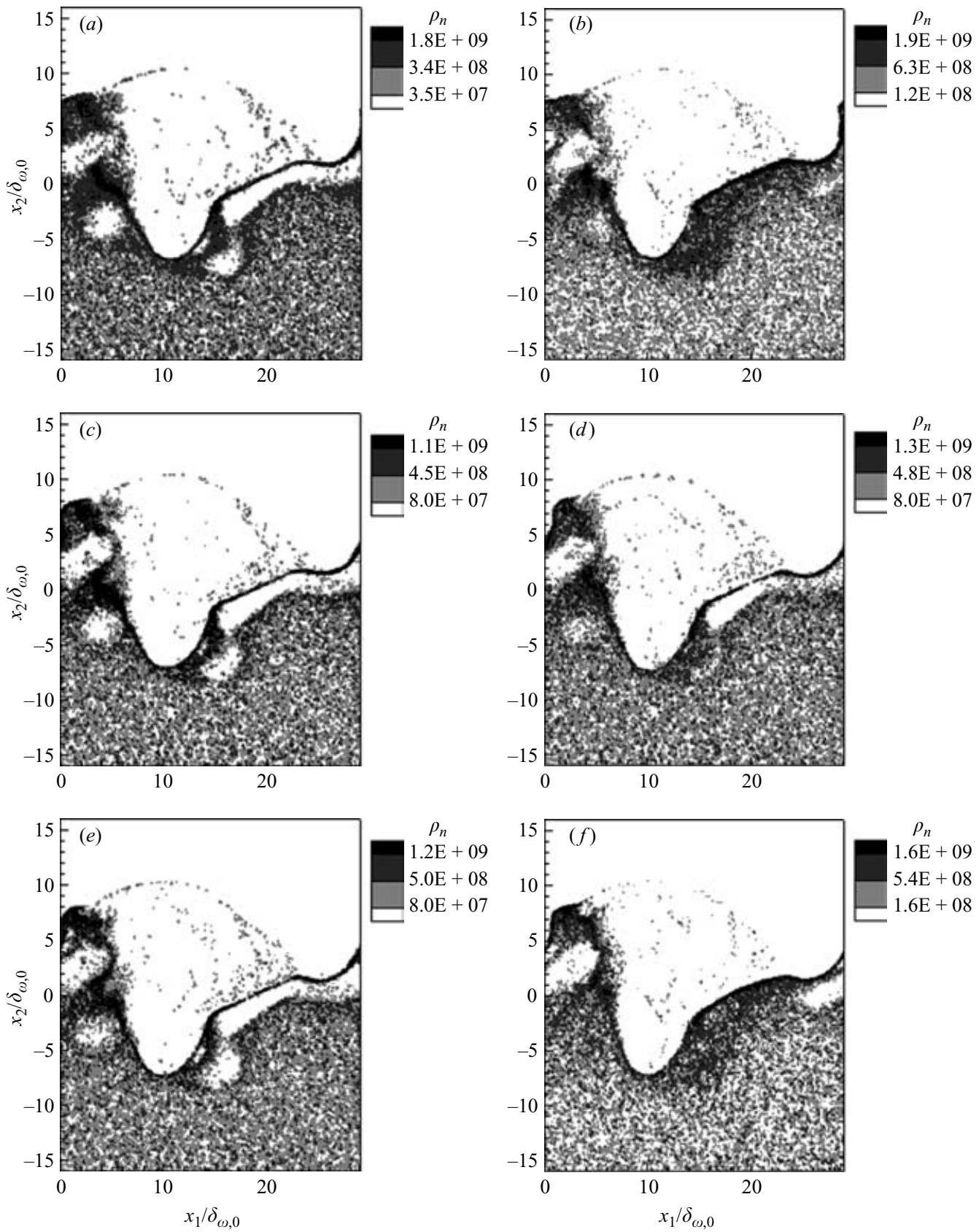

FiguRE 9. Contour plots of the drop number density in the between-the-braid plane $x_{3} / \delta_{\omega, 0}=$ 8.75 at $t^{*}=95$. MCdie375 (a), MCdie375x (b), MCdie400 (c), MCjetA400 (d), MCdie425 $(e)$, and MCrp1400 $(f)$.

the same $S t_{0}$ means that more drops interact with the flow which creates more local non-uniformities through the drag action; however, $\left\{\left\{D_{0}\right\}\right\}$ being slightly smaller, combined with the larger $T_{0}$ means that the drop lifetime is reduced, which decreases the drops/flow interaction time. Increasing $X_{v, 0}$ (figures $9 a$ and $9 b$ ) decreases the complexity of the drop organization, this being due to the opposite effect of the 
longer drop lifetime which increases the interaction with the flow and promotes non-uniformities, and of the flow difficulty in transporting a larger mass loading as the drops remain larger; apparently, the latter effect dominates at $t^{*}=95$. Finally, comparisons among diesel, Jet A and RP-1 (JP-7 contours are similar to the other kerosenes) in figures $9(a), 9(d)$ and $9(f)$, show the specific characteristics of the diesel drop distribution when compared to the kerosenes; some of these differences may occur because of the marginally smaller $N_{0}$ and $\left\{\left\{D_{0}\right\}\right\}$ for diesel at the same $S t_{0}$, and their specific effect is difficult to separate from the other influences on the flow. Generally, the kerosenes being more volatile than diesel, their faster drop evaporation leads to a less complex drop field.

\subsubsection{Flow field}

The vortical activity is first addressed, followed by the vapour distribution and composition.

To understand the impact of drop-liquid composition on the vortical aspect of the flow, contour plots of $\omega_{1}$ and $\omega_{3}$ were examined. Because both $\omega_{1}$ and $\omega_{3}$ show the same trends with varying liquid volatility, $T_{0}$ and $X_{v, 0}$, only $\omega_{3}$ will be here discussed. Contour plots of $\omega_{3}$ are illustrated in figure 10 for all fuels at $T_{0}=375 \mathrm{~K}$. As already stated, since $\omega_{3}$ is initially negative everywhere in the computational domain, the appearance of positive $\omega_{3}$ is an indication of small-scale production. Results from the transitional study of Le Clercq \& Bellan (2004) (their figure 6) show that flows with MC-liquid drops have more complex structure and decreased vortical activity compared to their SC-liquid counterpart. This reduction in vortical activity is conjectured to result from the higher longevity of the MC-liquid drops which promotes drop/flow interaction. This interaction was shown by Okong'o \& Bellan (2004) to dominate the dissipation, which is the mechanism that transforms organized flow activity (e.g. motion, energy) into the random motion that is essentially heat. Comparing the Okong'o \& Bellan (2004) velocity-fluctuation energy spectra of SC simulations (their figure 2) to the Le Clercq \& Bellan (2004) equivalent spectra of MC simulations (their figure 5), it is clear that in the $\mathrm{MC}$ case, the largest scales contain more energy and the smallest scales contain considerably less (three orders of magnitude) energy. This means that the Kolmogorov process of energy cascade is less effective in MC situations, consistent with the reduced small-scale activity evident in the Le Clercq \& Bellan (2004) MC simulations. Figure 10 shows that although the small-scale production is subdued, as expected, in this pre-transitional study compared to the simulations of Le Clercq \& Bellan (2004), the level of small-scale vortical activity is reduced for $\mathrm{MC}$ liquids that are less volatile than the SC liquid (diesel in figure $10 b$ compared to $\mathrm{n}$-decane in figure $10 a$ ) and is enhanced for MC liquids containing more volatile species than the SC fuel (Jet A in figure 10c compared to n-decane in figure 10a). Among MC liquids, the range of small-scale activity is directly correlated with the smallest molar weight species in the mixture and decreases from Jet A to diesel, and further to JP-7 and RP-1 (figures 10c, 10b, 10e and 10f), in complete accord with the PDFs displayed in figure 2. In fact, RP-1 and JP-7, which have overlapping PDFs in the smallest- $m$ range, also have almost identical ranges of $\omega_{3}$ values. Thus, there is a direct link between liquid composition and the development of the small-scale vortical activity in the flow.

For a specific liquid (here, diesel) the small-scale vortical activity is enhanced in magnitude at the larger $X_{v, 0}$, but is locally concentrated in smaller regions (compare figures $10 \mathrm{~b}$ and $10 \mathrm{~d}$ ). These small pockets of higher small-scale vortical activity occur in the low- $\rho_{n}$ regions at the edge of high- $\rho_{n}$ localities (compare figures 5 and 9). 

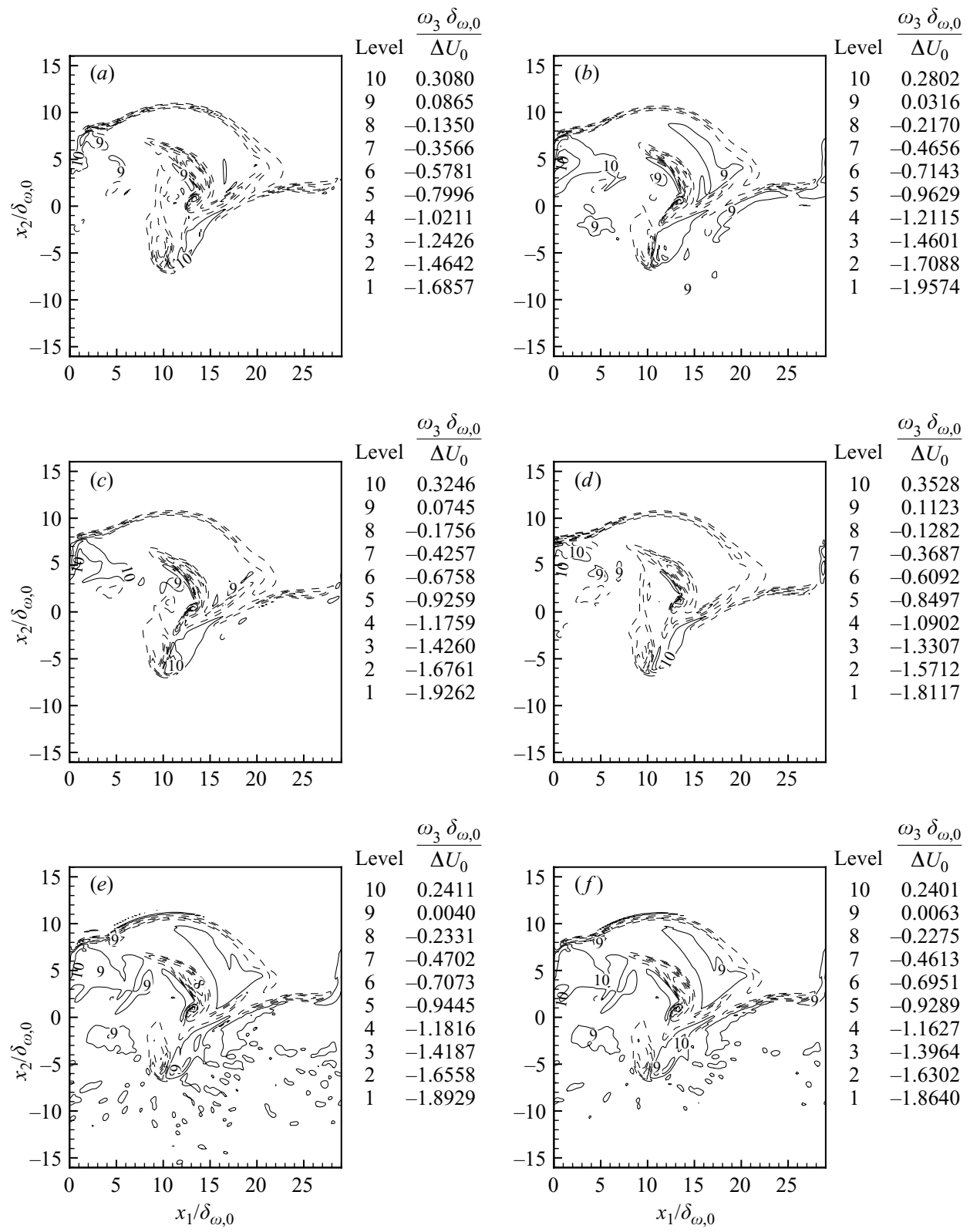

FIGURE 10. Contour plots of the spanwise vorticity in the between-the-braid plane $x_{3} / \delta_{\omega, 0}=$ 8.75 at $t^{*}=95$. SCdec375 (a), MCdie375 (b), MCjetA375 (c), MCdie375x (d), MCrp1375 $(e)$, and MCjp7375 $(f)$.

Consideration of the $T_{0}$ effect on the vortical activity shows that it is negligible in the range examined.

The $Y_{v}$ contours are shown in figure 11 for selected simulations. In all cases, the lower stream has the highest $Y_{v}$, the upper stream contains the smallest $Y_{v}$ and the mixing layer displays a very inhomogeneous mixture. Compared to n-decane (figure 11a), diesel (figure 11b) produces a much smaller $Y_{v}$, with a maximum value that is less 

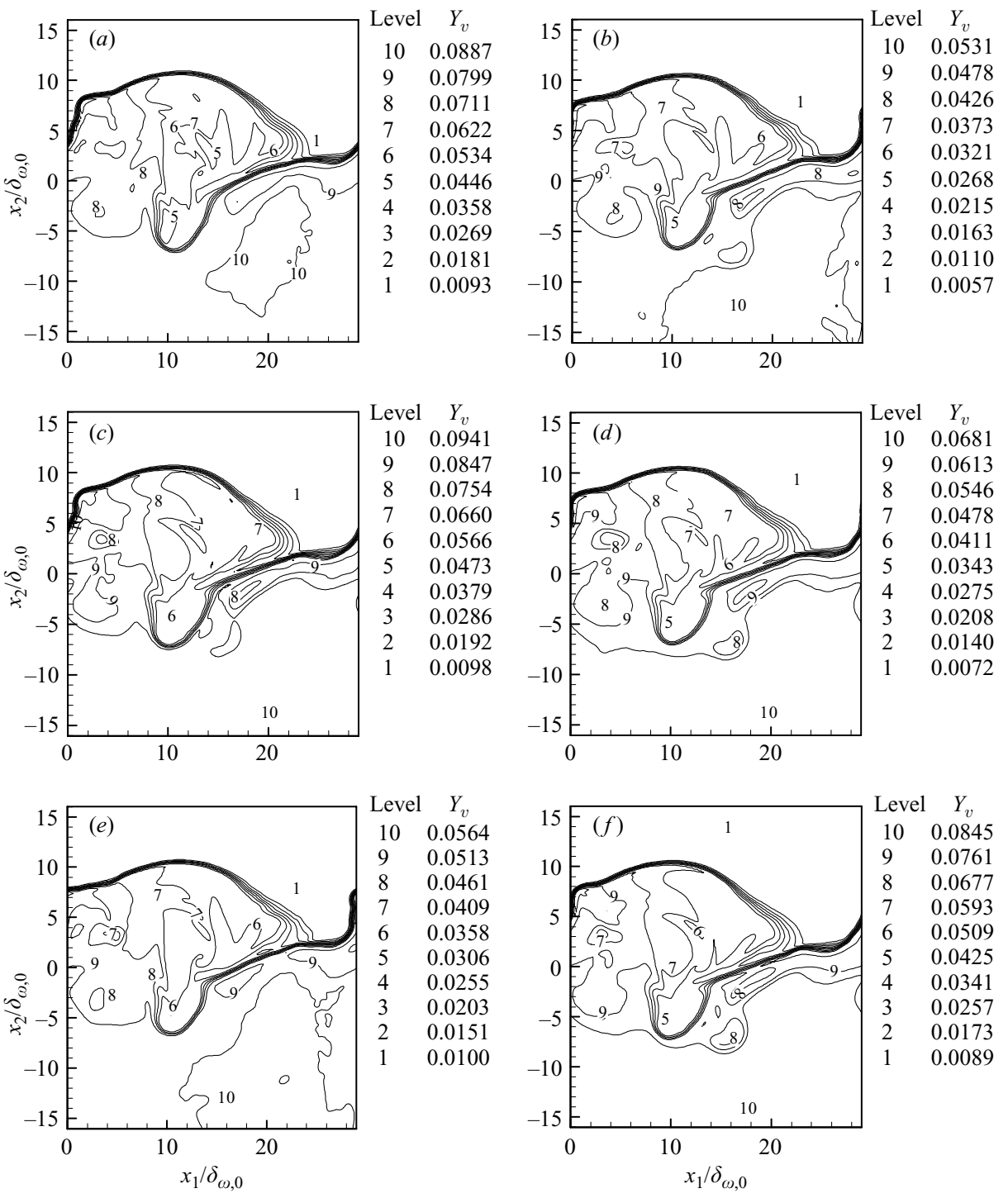

FIGURE 11. Contour plots of the vapour mass fraction in the between-the-braid plane $x_{3} / \delta_{\omega, 0}=$ 8.75 at $t^{*}=95$. SCdec375 (a), MCdie375 (b), MCjetA400 (c), MCdie400 (d), MCdie375x $(e)$, and MCdie425 $(f)$.

than $60 \%$ that for n-decane ( $\rho$ values are similar). With increasing $T_{0}$ (figures $11 b$, $11 d$ and $11 f$ ), the layer entrains hotter gas, and the promoted evaporation increases $Y_{v}$. Jet A (figure 11c) being more volatile than diesel, the corresponding maximum $Y_{v}$ is larger, representing the trend for all kerosenes (not shown). Finally, as $X_{v, 0}$ increases, the maximum $Y_{v}$ is larger, this being happenstance and representing the opposite effect of larger $Y_{v, 0}$ combined with slower evaporation. For SC liquids, the $Y_{v}$ information completes the state of the vapour; however, for MC liquids the composition is necessary to entirely characterize the vapour.

Mixture composition contours of MC liquids are displayed in figure 12 for $\theta_{v}$ and figure 13 for $\sigma_{v}$. Independent of the simulation, both $\theta_{v}$ and $\sigma_{v}$ exhibit strong spatial 

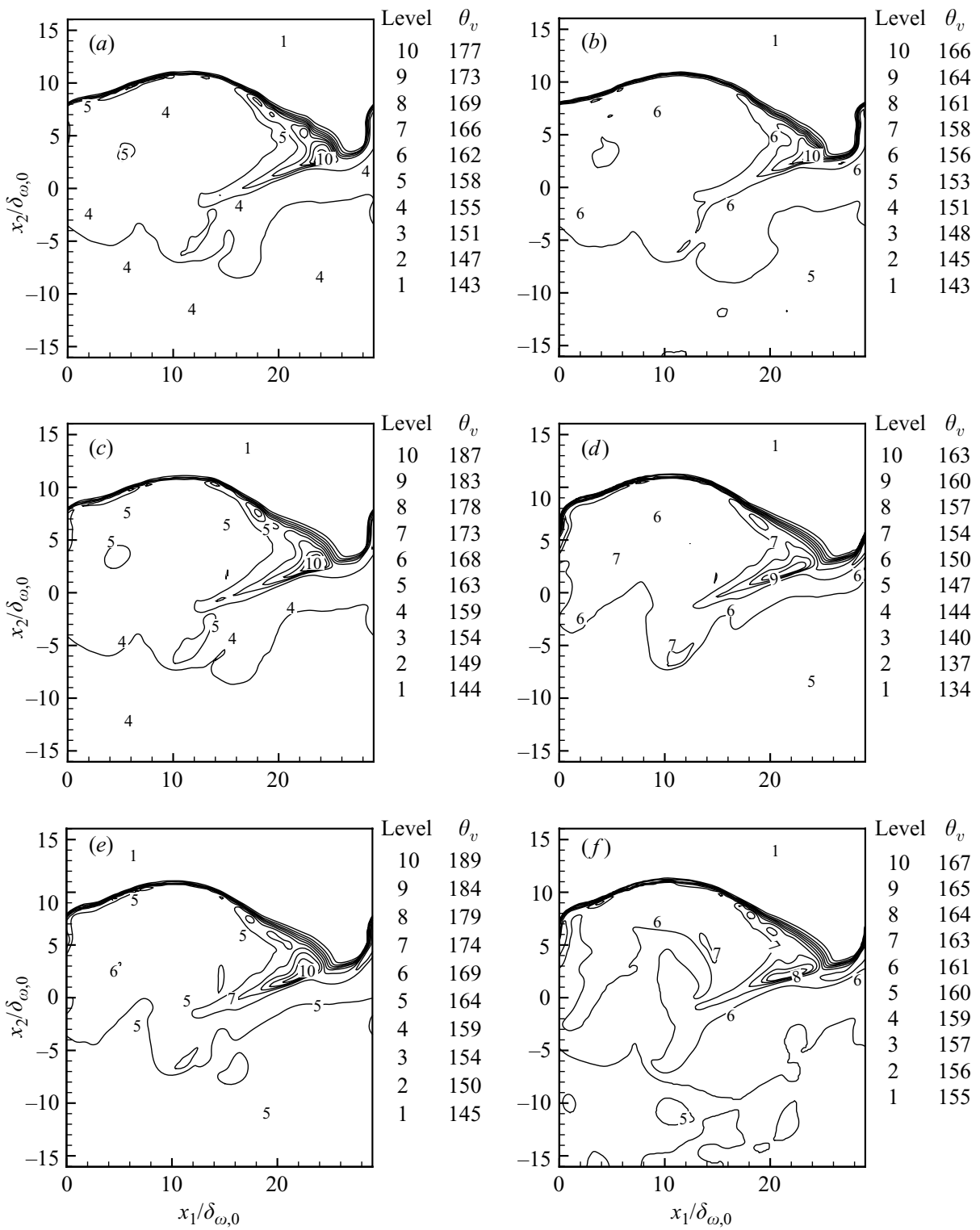

FIGURE 12. Contour plots of the vapour mean molar mass $\left(\mathrm{kg} \mathrm{kmole}^{-1}\right)$ in the between-thebraid plane $x_{3} / \delta_{\omega, 0}=8.75$ at $t^{*}=95$. MCdie375 (a), MCdie375x (b), MCdie400 (c), MCjetA400 $(d), \operatorname{MCdie} 425(e)$, and MCrp1400 $(f)$.

inhomogeneities. For diesel at $T_{0}=375 \mathrm{~K}$ with $X_{v, 0}=10^{-4}$, the smallest $\theta_{v}$ value is in the upper stream and corresponds to the initial condition. The next larger values are in the lower stream because this is where the drops initially reside and thus where the more volatile species evaporate. The mixing layer contains the intermediate-molarmass species which evaporate only after the drops have already been entrained into the layer. The largest $\theta_{v}$ value is within the layer at the location of the highest $\rho_{n}$ (e.g. compare figures $12 a$ and $9 a$ ) because the heaviest-molar-mass species are the least volatile and evaporate only after the drop-field structure has been established. 

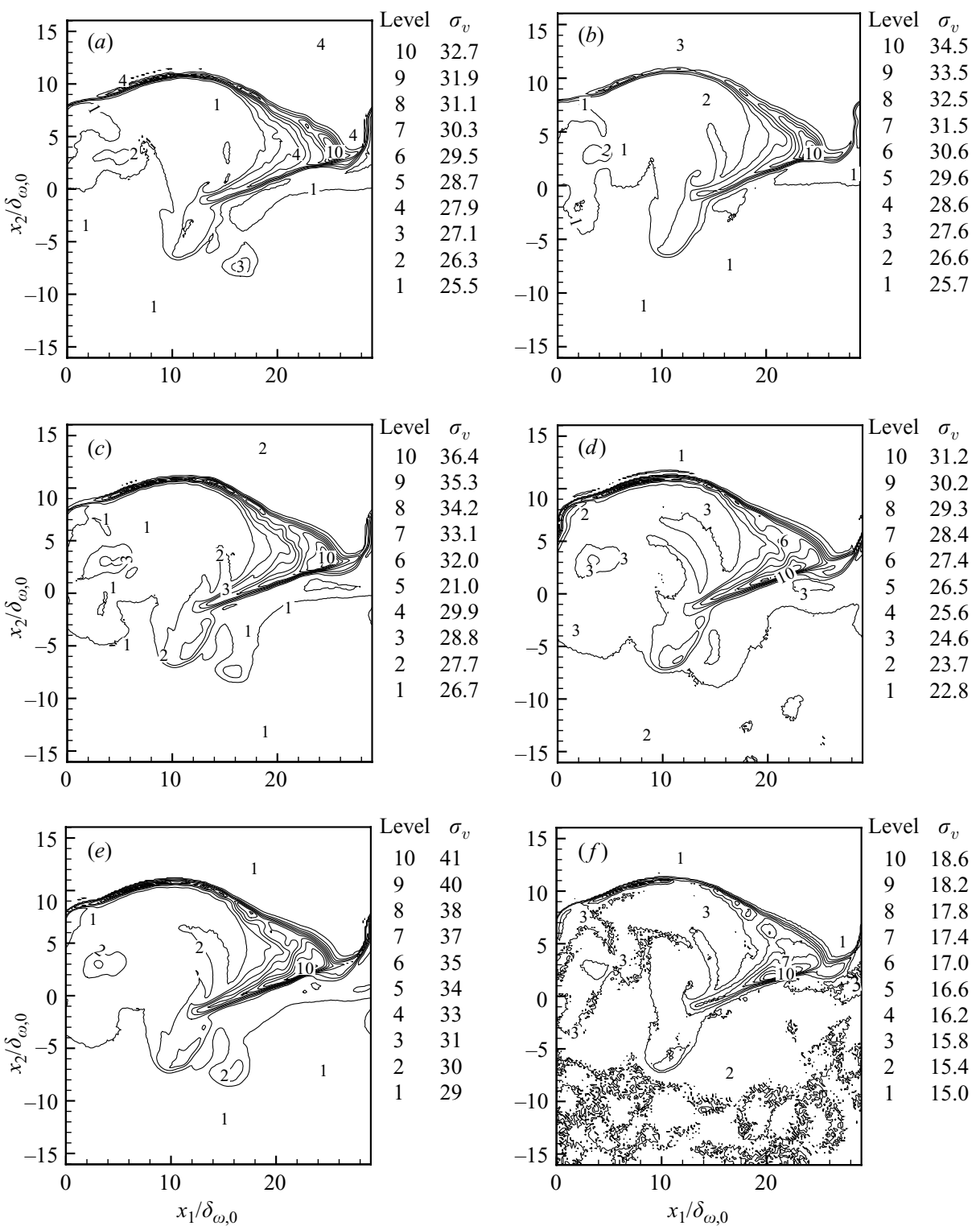

FIGURE 13. Contour plots of the vapour composition standard deviation $\left(\mathrm{kg} \mathrm{kmole}^{-1}\right)$ in the between-the-braid plane $x_{3} / \delta_{\omega, 0}=8.75$ at $t^{*}=95$. MCdie375 $(a), \operatorname{MCdie} 375 \mathrm{x}(b), \operatorname{MCdie} 400$ $(c), \operatorname{MCjetA} 400(d), \operatorname{MCdie} 425(e)$, and MCrp1400 $(f)$.

The smallest $\sigma_{v}$ is in the lower stream and also within the layer where $\rho_{n}$ is small, or where $\rho_{n}$ is large but $\left\{\left\{D_{0}\right\}\right\}$ is (conjectured) small, indicating that most species have evaporated. Intermediate values of $\sigma_{v}$ are found in the upper stream where species from the layer may be transported, changing the initially uniform composition, and also encountered in the mixing layer adjacent to the location of the largest $\rho_{n}$ because the heaviest evaporating species transported from the largest $\rho_{n}$ location will have a relatively large impact on $\sigma_{v}$. The largest $\sigma_{v}$ is at the location of the largest $\rho_{n}$ where the heaviest species evolve from the drops. Thus, a composition stratification 
is established, much as had been observed for laminar flow combustion with two species (Continillo \& Sirignano 1988), where those one-dimensional simulations were performed with computational instead of physical drops. Information not available in those binary-species computations is presented by $\sigma_{v}$ which exhibits strong local variations, particularly in the regions of highest $\theta_{v}$.

With increasing $X_{v, 0}$, the maximum value of $\theta_{v}$ decreases (figures $12 a$ and $12 b$ ) and that of $\sigma_{v}$ increases (figures $13 a$ and $13 b$ ) indicating a reduced evaporation rate which did not allow the evolution of the heavier species observed at the lower $X_{v, 0}$, where a smaller variation of the composition is observed as manifested by the smaller $\sigma_{v}$. When $T_{0}$ is larger, the maximum $\theta_{v}$ increases (figures $12 a, 12 c$ and $12 e$ ) and the range of $\sigma_{v}$ is augmented (figures $13 a, 13 c$ and 13e) because the larger evaporation rate allows increasingly heavier species to evolve from the drops, which increases $\sigma_{v}$. Finally, at the same $T_{0}$, both $\theta_{v}$ and $\sigma_{v}$ are liquid specific. The narrower PDF of Jet A, shifted to the lower- $m$ regime with respect to diesel, results in smaller maximum $\theta_{v}$ (figures $12 c$ and $12 d$ ) and a shifted range of $\sigma_{v}$ towards lower values (figures $13 c$ and $13 d$ ); a more non-uniform distribution of $\sigma_{v}$ is also apparent. For RP-1, its narrower PDF with respect to both diesel and Jet A leads to a decreased range for both $\theta_{v}$ (figures $12 c, 12 d$ and $12 f$ ) and $\sigma_{v}$ (figures $13 c, 13 d$ and $13 f$ ). For JP-7 (not shown), its wider PDF with respect to RP-1 but narrower than Jet A leads both $\theta_{v}$ and $\sigma_{v}$ to respective ranges of values intermediary to those of the two other kerosenes. Thus, the composition of the vapour is distinct for each of the liquids and the trends can be directly traced to the original composition. This information is inherently unavailable in SC simulations, and therefore caution must be exercised in using SC liquids as surrogates for MC liquids, either in simulations or in experiments.

\subsection{Homogeneous-plane averages}

\subsubsection{Dynamics}

To explore the reasons for the amplification in gas vortical activity with increased high-volatility species in the liquid, the budgets of the conservation equations for $\omega$ and $\omega \cdot \omega$

$$
\begin{gathered}
\frac{\mathrm{D} \boldsymbol{\omega}}{\mathrm{D} t}=(\boldsymbol{\omega} \cdot \nabla) \boldsymbol{u}-(\nabla \cdot \boldsymbol{u}) \boldsymbol{\omega}-\nabla\left(\frac{1}{\rho}\right) \times \nabla p+\nabla \times\left(\frac{1}{\rho} \nabla \cdot \boldsymbol{\tau}\right) \\
+\nabla \times\left(\frac{1}{\rho} \boldsymbol{S}_{\mathrm{mom}}\right)-\nabla \times\left(\frac{1}{\rho} S_{\text {mass }} \boldsymbol{u}\right), \\
\frac{\mathrm{D}(\boldsymbol{\omega} \cdot \boldsymbol{\omega})}{\mathrm{D} t}=2 \boldsymbol{\omega} \cdot(\boldsymbol{\omega} \cdot \nabla \boldsymbol{u})-2(\nabla \cdot \boldsymbol{u}) \boldsymbol{\omega} \cdot \boldsymbol{\omega}-2 \boldsymbol{\omega} \cdot\left[\nabla\left(\frac{1}{\rho}\right) \times \nabla p\right]+2 \boldsymbol{\omega} \cdot\left[\nabla \times\left(\frac{1}{\rho} \nabla \cdot \boldsymbol{\tau}\right)\right] \\
+2 \boldsymbol{\omega} \cdot\left[\nabla \times\left(\frac{1}{\rho} \boldsymbol{S}_{\mathrm{mom}}\right)\right]-2 \boldsymbol{\omega} \cdot\left[\nabla \times\left(\frac{1}{\rho} S_{\text {mass }} \boldsymbol{u}\right)\right],
\end{gathered}
$$

were assessed, where $\mathrm{D} / \mathrm{D} t$ is the substantial derivative. Illustrated in figure 14 are the $\left(x_{1}, x_{3}\right)$ homogeneous-plane-average budget RMS of $\omega_{1}$ and $\omega_{3}$ at $t^{*}=95$ for the diesel and Jet $A$ simulations at $T_{0}=375 \mathrm{~K}$, and for diesel at $T_{0}=425 \mathrm{~K}$. For both $\omega_{1}$ and $\omega_{3}$, and irrespective of the simulation, the maximum contribution is that of the stretching and tilting term, followed by the stresses and then by $\boldsymbol{S}_{\text {mom }}$, with all other terms being negligible. For $\omega_{1}, S_{\text {mom }}$ contributes throughout the entire layer, with similar magnitude peaks in the lower and middle part of the layer, and a larger peak in the upper layer region. For $\omega_{3}$, all three significant terms have similar contributions except in the middle of the layer and in its upper part where 

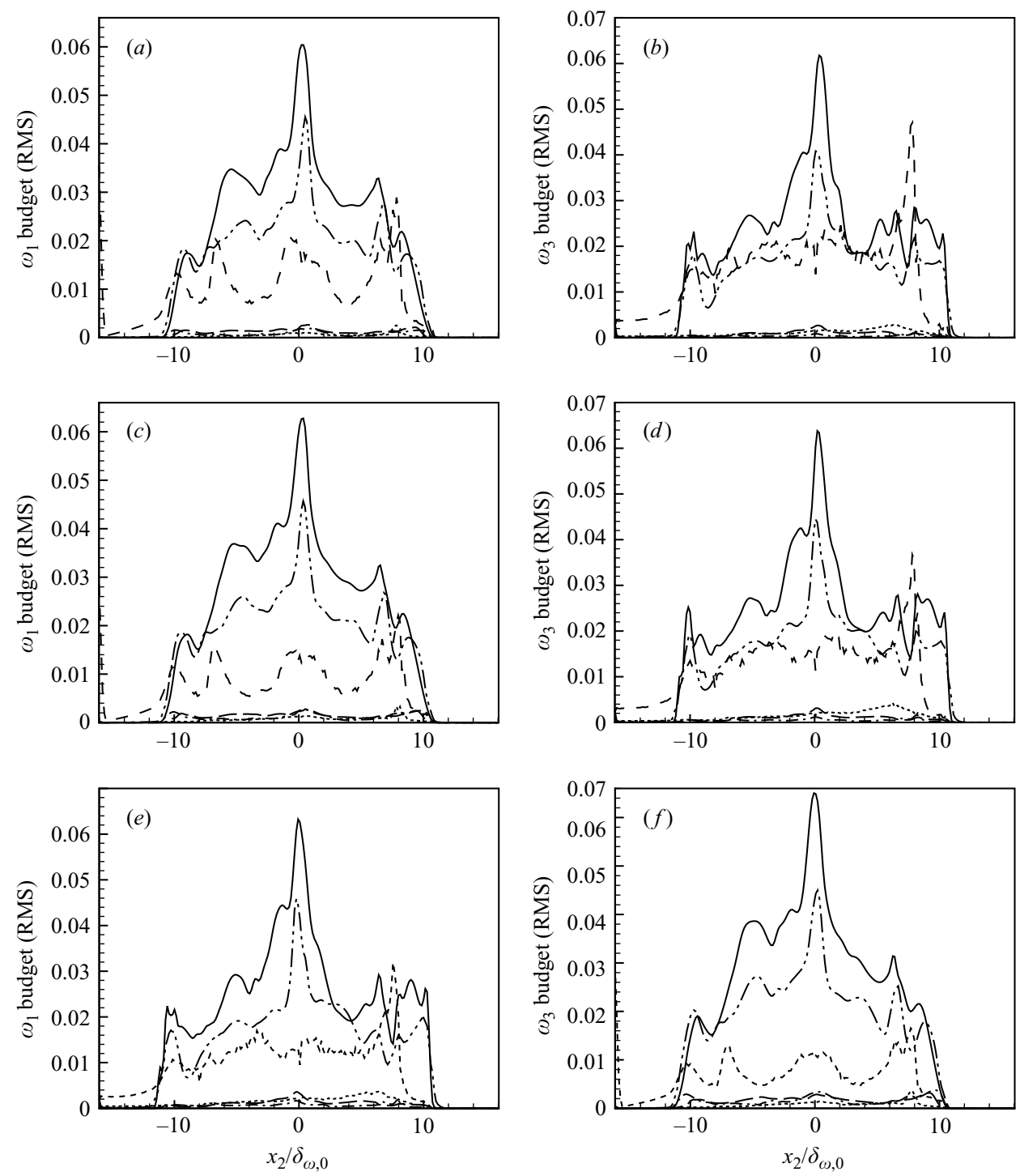

FIGURE 14. Homogeneous $\left(x_{1}, x_{3}\right)$-plane-average streamwise vorticity budget $(a, c, e)$ and spanwise vorticity budget $(b, d, f)$ at $t^{*}=95$ for three simulations: $(a, b)$ MCdie 375, $(c, d)$ MCjetA375 and $(e, f)$ MCdie425. -,$(\omega \cdot \nabla) u /\left(\Delta U_{0} / \delta_{\omega, 0}\right)^{2} ;---,(\nabla \cdot u) \omega /\left(\Delta U_{0} / \delta_{\omega, 0}\right)^{2}$; $-\cdot-\cdot, \quad \nabla(1 / \rho) \times \nabla p /\left(\Delta U_{0} / \delta_{\omega, 0}\right)^{2} ;-\cdot \cdot-\cdot \cdot-, \nabla \times(1 / \rho \nabla \cdot \tau) /\left(\Delta U_{0} / \delta_{\omega, 0}\right)^{2} ;---, \nabla \times\left(1 / \rho S_{\text {mom }}\right) /$ $\left(\Delta U_{0} / \delta_{\omega, 0}\right)^{2} ; \cdots, \nabla \times\left(1 / \rho S_{\text {mass }} u\right) /\left(\Delta U_{0} / \delta_{\omega, 0}\right)^{2}$.

$S_{\text {mom }}$ exhibits a dominating sharp peak. Visual correlations show that all $S_{\text {mom }}$ peaks occur in the regions of highest $\rho_{n}$ observed in figure 9. To elucidate the phenomenon responsible for the magnitude of $\boldsymbol{S}_{\text {mom }}$, this term is decomposed as

$$
\begin{gathered}
\boldsymbol{S}_{\text {mom }}=-\sum_{q=1}^{N} \frac{w_{q}}{\Delta V_{q}}\left[\boldsymbol{F}+\left(\theta_{l} \frac{\mathrm{d} \mathscr{N}}{\mathrm{d} t}+\mathscr{N} \frac{\mathrm{d} \theta_{l}}{\mathrm{~d} t}\right) \boldsymbol{v}\right]_{q} \\
=\boldsymbol{S}_{\text {mom,drag }}+\boldsymbol{S}_{\text {mom,evap }}+\boldsymbol{S}_{\text {mom,mean }}
\end{gathered}
$$


Budgets of (3.6) (not illustrated) show the preponderant term to be $\boldsymbol{S}_{\text {mom,drag }}$, indicating that the source term in the budgets of (3.4) and (3.5) is mostly of dynamic nature for these conditions. With increasing volatility and/or $T_{0}$, the drops are smaller at $t^{*}=95$ due to enhanced evaporation, resulting in a smaller drag. The smaller contribution at $t^{*}=95$ of $S_{\text {mom }}$ to the vorticity with increasing volatility and/or $T_{0}$ is more than compensated by the increase in the stretching and tilting effect which allows an augmented vortical activity of the flow. The increase in the tilting and stretching effect is partially due to the reduced drag which decreases the dissipation, as shown by Okong'o \& Bellan (2004), and permits the retention of more organized vorticity. Although for the conditions of the present simulations $\boldsymbol{S}_{\text {mom,evap }}$ and $\boldsymbol{S}_{\text {mom,mean }}$ are small compared to $S_{\text {mom,drag }}$, one can conceive of situations where the liquid is more volatile with respect to the flow characteristic time, so that the rate of change in drop composition is substantial and leads the vorticity evolution of the flow. The potential of substantial thermodynamic contributions to the vorticity from these evaporative and composition sources indicates intriguing dynamics/composition strong coupling possibilities in these compressible flows.

Similar to the contour plots, the differences between the simulations with diesel and Jet $\mathrm{A}$ are smaller, but noticeable in this pre-transitional study. For $\omega_{1}$, the Jet A results (figure 14c) show enhanced stretching and tilting effects compared to those for diesel (figure 14a) and a reduced activity of the $S_{\text {mom }}$ term, particularly in proximity to the upper stream. For $\omega_{3}$ (figures $14 b$ and $14 d$ ), the same differences are observed as for $\omega_{1}$ and additionally, the more dramatic reduction in the $\boldsymbol{S}_{\text {mom }}$ contribution for Jet A close to the upper stream is accompanied by a slightly augmented contribution of the viscous stresses. Comparing the diesel results at different $T_{0}$, one observes for $\omega_{1}$ that at the higher $T_{0}$ the stretching and tilting term has larger value peaks, the viscous contribution is more uniform across the layer (reduced in the middle and enhanced elsewhere) and $\boldsymbol{S}_{\text {mom }}$ is additionally reduced compared to Jet A. Examining $\omega_{3}$ for the diesel simulations at the higher $T_{0}$, one observes the same differences detected between diesel and Jet A, but now exacerbated. Thus, the liquid composition mostly affects the $\boldsymbol{S}_{\text {mom }}$ term, then to a smaller extent the stretching and tilting term, which is related to enstrophy production, and minimally the viscous stresses. Because both higher volatility and larger $T_{0}$ have qualitatively the same effect, in that they promote evaporation, the $\omega \cdot \omega$ budget is addressed next only in the context of the $T_{0}$ variation.

Depicted in figure 15 are the $\left(x_{1}, x_{3}\right)$ homogeneous-plane-average budget RMS of $\omega \cdot \omega$ at $t^{*}=95$ for the diesel simulations at $T_{0}=375 \mathrm{~K}$ and $425 \mathrm{~K}$. Generally, the stretching and tilting term and the stresses almost equally contribute in most of the layer; all other terms of (3.5) are negligible. An exception is for $T_{0}=375 \mathrm{~K}$ where at the highest $\rho_{n}$ location in the upper part of the layer, $\boldsymbol{S}_{\mathrm{mom}}$ is larger than the two other significant terms. Consistent with the information provided by figure 14 , the effect of promoted evaporation is to enhance the stretching and tilting and the viscous contribution, and to depress the $S_{\text {mom }}$ apport.

\subsubsection{Vapour composition and temperature}

Homogeneous-plane averages of $\theta_{v} / \theta_{v, 0}$ and $\sigma_{v} / \sigma_{v, 0}$ at $t^{*}=95$ are displayed in figures $16(a)$ and $16(b)$ along with the degree of departure from the SGPDF embodied by $\varepsilon_{v}$ in figure $16(c)$, and the normalized $\langle T\rangle$ in figure $16(d)$. Since no equation was solved for $\sigma_{v}$, the ordinate label of figure $16(b)$ is a reminder that it was calculated as $\sigma_{v}=\sqrt{\left(\psi_{v}-\theta_{v}^{2}\right)}$. Liquid-specific variations are evident, although for all liquids the vapour is contained within the mixing layer and the lower stream; the upper stream composition is unchanged from the initial one. The lower stream uniform $\left\langle\theta_{v}\right\rangle / \theta_{v, 0}$ 

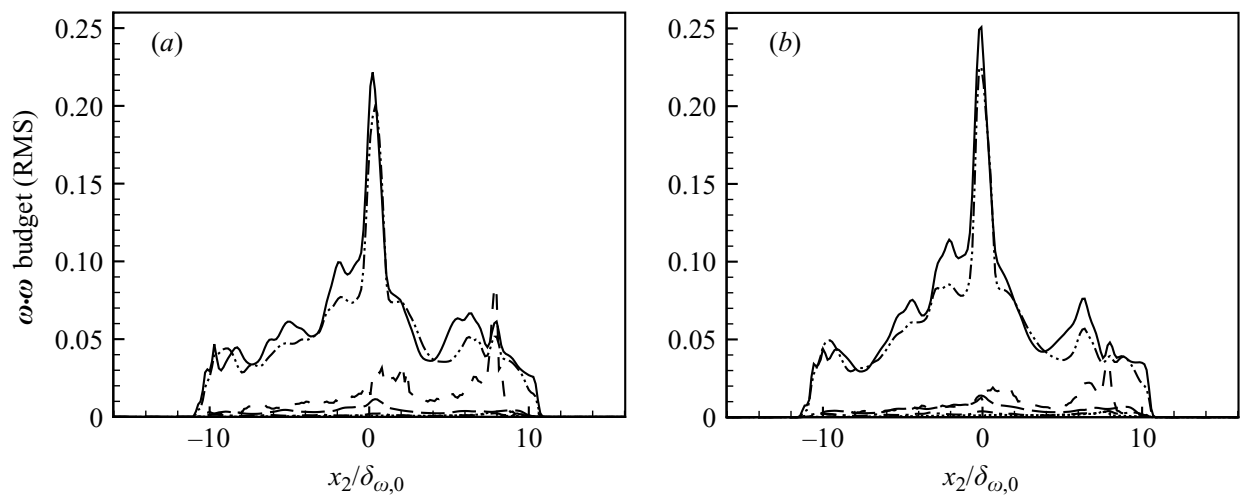

FIgURE 15. Homogeneous $\left(x_{1}, x_{3}\right)$-plane-average $\omega \cdot \omega$ budget at $t^{*}=95$ for two simulations: (a) MCdie375 and $(b)$ MCdie425. —, $2 \omega \cdot((\omega \cdot \nabla) u) /\left(\Delta U_{0} / \delta_{\omega, 0}\right)^{3} ;---, 2 \omega^{2} \cdot(\nabla \cdot u) /$ $\left(\Delta U_{0} / \delta_{\omega, 0}\right)^{3} ;-\cdot-\cdot-, 2 \omega \cdot(\nabla(1 / \rho) \times \nabla p) /\left(\Delta U_{0} / \delta_{\omega, 0}\right)^{3} ;-\cdot \cdot-, 2 \omega \cdot(\nabla \times(1 / \rho \nabla \cdot \tau)) /\left(\Delta U_{0} / \delta_{\omega, 0}\right)^{3} ; \cdots \cdots$, $2 \omega \cdot\left(\nabla \times\left(1 / \rho S_{\text {mass }} u\right)\right) /\left(\Delta U_{0} / \delta_{\omega, 0}\right)^{3}$.
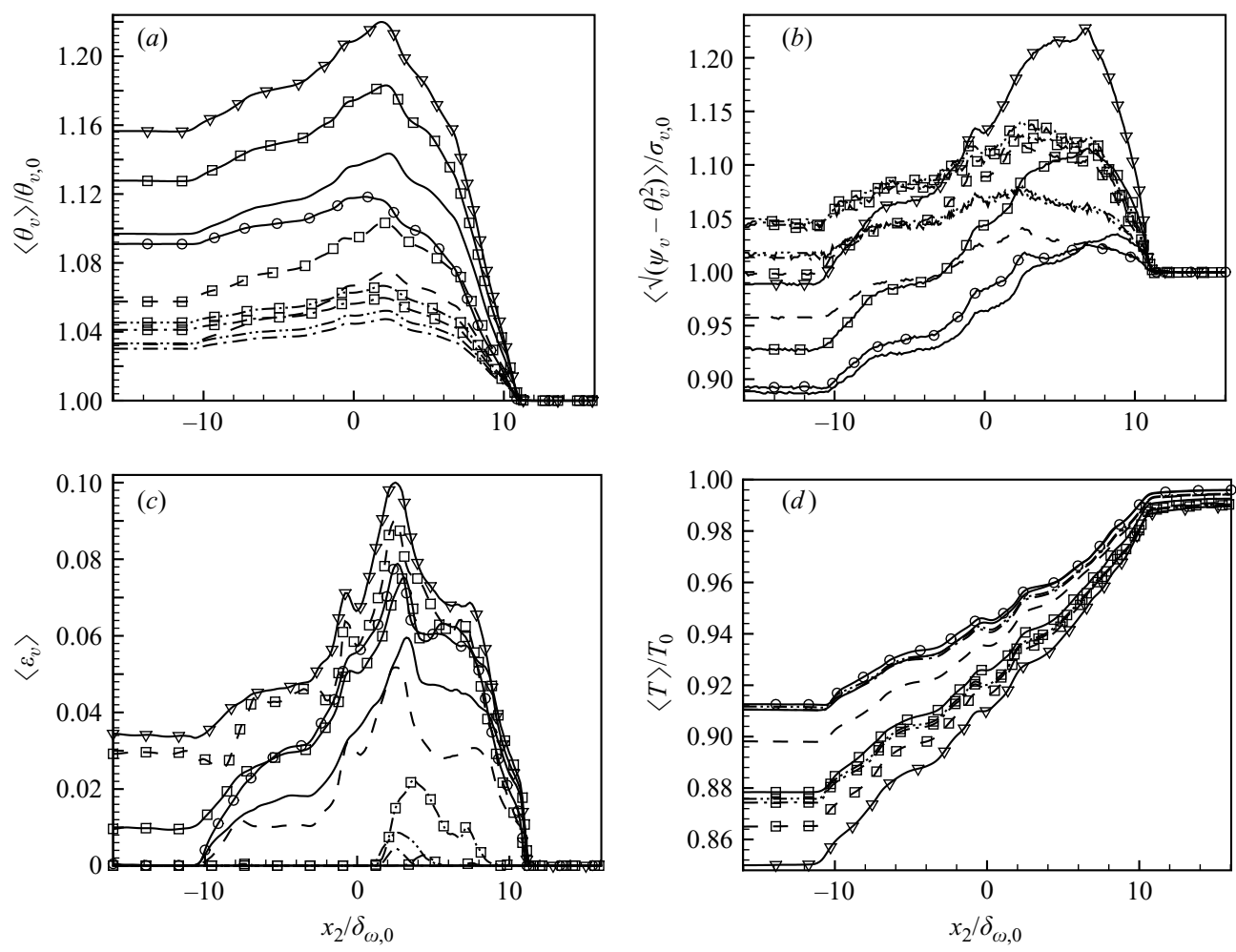

FIGURE 16. Homogeneous-plane averages at $t^{*}=95$ of the normalized vapour mean molar mass $(a)$, the normalized standard deviation $(b)$, the parameter $\varepsilon_{v}(c)$ and, the normalized gas temperature $(d)$. - MCdie 375; —o—o-, MCdie 375x; - $\square-$, MCdie $400 ;-\nabla-$, MCdie425; - - - , MCjetA375; - $\square-\square-$, MCjetA400; -.--, MCrp1375; - $-\square-$ - MCpr1400; -..-.-, MCjp7375;--๑-•๑-, MCjp7400.

and $\left\langle\sigma_{v}\right\rangle / \sigma_{v, 0}$ is consistent with the drop saturation discussed above and with the constant $\langle T\rangle$. Among all liquids, diesel evaporation produces the largest change in $\left\langle\theta_{v}\right\rangle / \theta_{v, 0}$, mainly due to its very wide species distribution, followed by Jet A, JP-7 
and RP-1, this ordering being entirely consistent with the width of the initial PDF shown in figure 2. Of all kerosenes, Jet A produces the largest $\left\langle\theta_{v}\right\rangle / \theta_{v, 0}$ in the mixing layer, consistent with the wider PDF on the high- $m$ side, and relatively smaller values in the lower stream, consistent with the wider PDF on the low- $m$ side. The $\left\langle\theta_{v}\right\rangle / \theta_{v, 0}$ profiles increase from the lower stream, peak and finally decline, more sharply with increasing $T_{0}$, to unity in the upper stream; the $\left\langle\theta_{v}\right\rangle / \theta_{v, 0}$ peak coincides with the contour-plot-identified regions of high $\theta_{v}$. For larger $X_{v, 0},\left\langle\theta_{v}\right\rangle / \theta_{v, 0}$ is reduced, and the largest reduction is in the mixing layer where most of the medium- $m$ species are released rather than in the lower stream where the small- $m$ species evaporate from the drops. The variation of $\left\langle\theta_{v}\right\rangle / \theta_{v, 0}$ with $T_{0}$ is small for JP-7 and RP-1, mild for Jet $A$ and substantial for diesel for which the peak for $T_{0}=425 \mathrm{~K}$ is almost $22 \%$, a value exceeding the maximum identified from the volume average presented in figure 7. In the lower stream, $\left\langle\sigma_{v}\right\rangle / \sigma_{v, 0}$ has decreased below unity in all diesel simulations, indicating a narrower PDF at $t^{*}=95$ which is due to condensation of the lighter vapour species onto the drops. Only JP-7 and RP-1 vapor escape this depletion of the lighter species at all $T_{0}$, with evaporation increasingly prevailing with higher $T_{0}$. The mixing layer is the site of substantial change in $\left\langle\sigma_{v}\right\rangle / \sigma_{v, 0}$, which increases approximately $25 \%$ across the layer for diesel at $T_{0}=425 \mathrm{~K}$, with smaller non-uniformities for Jet A and only mild ones for PR-1 and JP-7. The reduced variation of RP-1 and JP-7 with $T_{0}$ compared to Jet $\mathrm{A}$ is representative of their narrower initial PDF.

The departure of the gas composition PDF from the initial SGPDF is illustrated in figure $16(c)$ by the distribution of $\varepsilon_{v}$. In the upper stream, the PDF remains decisively a SGPDF for all simulations. Independent of the liquid or of the simulation conditions, the largest departure from the SGPDF occurs slightly in the upper part of the layer with respect to its middle, and visually correlates with the regions of high $\rho_{n}$ identified in figure 9 and with the peak in $\left\langle\theta_{v}\right\rangle / \theta_{v, 0}$. In this region, by $t^{*}=95$, the drops release only the higher- $m$, less volatile species, which mix with the medium- $m$ species previously released in the layer and with the small- $m$ species entrained from the lower stream to produce a very complex mixture. Finally, in the lower stream, $\varepsilon_{v}$ is null or small and uniform, indicating, consistent with $\left\langle\theta_{v}\right\rangle / \theta_{v, 0}$ and $\left\langle\sigma_{v}\right\rangle / \sigma_{v, 0}$, an equilibration in activity. For either RP-1 or JP-7 at $T_{0}=375 \mathrm{~K}$, there is negligible evolution of a second PDF peak, and although this second peak is slightly higher for RP-1 at $T_{0}=400 \mathrm{~K}$, it remains minimal. In the lower part of the mixing layer, the Jet A PDF is almost as double-peaked as that of diesel; however, due to the smaller $\rho_{n}$ of Jet $\mathrm{A}$ in the upper part of the mixing layer, there is less condensation, which leads to a smaller $\varepsilon_{v}$. With increased $T_{0}$, the DGPDF aspect is enhanced and reaches the largest $\varepsilon_{v}$ values for diesel at $T_{0}=425 \mathrm{~K}$. At $T_{0}=400 \mathrm{~K}$, Jet A assumes a more pronounced DGPDF aspect than diesel, reversing the trend at $T_{0}=375 \mathrm{~K}$ and indicating higher condensation activity. Finally, the larger $X_{v, 0}$ also leads to a more pronounced DGPDF, as there is a higher quantity of initially available lower- $m$ species to condense onto the drops.

The $\langle T\rangle$ distribution across the mixing layer is portrayed in figure $16(d)$. In the upper stream, $\langle T\rangle$ is uniform and close to $T_{0}$ since the temperature is reduced only by transport of heat resulting from gas entrained into the layer. In contrast, the lower stream temperature, also uniform, is reduced by about $9 \%$ to $15 \%$, depending on the simulation, this being the consequence of heat transfer to the drops. Between the lower and upper streams, the mixing layer $\langle T\rangle$ monotonically increases. The smallest reduction in $\langle T\rangle$ from $T_{0}$ is for the two diesel simulations at $T_{0}=375 \mathrm{~K}$ and for RP-1 and JP-7, also at $T_{0}=375 \mathrm{~K}$, with profiles that all coincide. For Jet A at $T_{0}=375 \mathrm{~K}$, 
the $\langle T\rangle$ reduction is slightly larger than for diesel and the other two kerosenes owing to its larger $L_{v}$ which means that increasing heat is removed from the flow to achieve evaporation. The larger $L_{v}$ is due to lower- $m$ species contained in the mixture when compared to diesel and the other two kerosenes (see figure 2, (2.16) and (2.17)). At larger $T_{0},\langle T\rangle$ is further diminished as evaporation is more vigorous and requires increased heat transfer from the gas phase. The $\langle T\rangle$ distribution is independent of $X_{v, 0}$ because $N_{0}$ and $\left\{\left\{D_{0}\right\}\right\}$ are the initial conditions that determine heat transfer from the gas to the drops.

\section{Conclusions}

A formulation describing the composition of multicomponent fluids as a linear combination of two Gamma probability distribution functions (PDFs) depending on the molar mass has been used in conjunction with PDF-consistent conservations equations to describe many liquid drops evaporating in a gas flow. Each drop is followed in a Lagrangian frame and its trajectory, mass, momentum, energy and composition change as a result of its interaction with the flow. The gas is followed in an Eulerian frame and source terms in the conservation equations account for the impact of the drops on the flow. Both liquid and gas composition are described by the same mathematical form of the PDF, but with parameters that change temporally and with each drop for the liquid, and temporally and with location for the gas. This model has been applied to investigate the drop/flow interaction between evaporating drops in a three-dimensional temporal mixing layer whose lower stream, containing a carrier gas, is initially laden with a collection of randomly distributed polydiperse-size drops. The layer initially consists of four spanwise vortices whose double pairing, promoted by a perturbation, results in the formation of an ultimate vortex. Comparisons were made among simulations performed with a single chemical species (n-decane), diesel and three kerosenes (Jet A, RP-1 and JP-7). The initial conditions, which could not all be matched, focused on the same mass loading, Reynolds number and drop/flow interaction through the specification of the Stokes number, all being non-dimensional numbers that allow comparisons of self-similar solutions in the spirit of Batchelor (1967). The initial liquid composition was chosen to consist of a single Gamma PDF, so as to detect the evolution of the second Gamma PDF which is indicative of condensation onto the drops.

The results have been analysed to study the evolution of the layer and drops, as well as the state obtained at the highest momentum thickness of the layer. The layer growth and global vortical aspects of the flow were shown to be unaffected by the identity of the liquid; however, a measure of the global mixing was highly liquidspecific and additionally varied with the carrier gas temperature and extent of initial vapour vitiation of the free-stream gas. Also liquid-specific, determined by the initial liquid volatility, were the evolution of ensemble-average drop temperature, size, mass and composition. The liquid saturation temperature was shown to increase for all liquid mixtures and the ratio of the drop temperature to the saturation temperature displayed a complex variation according to the simulation, depending on both the liquid identity and on the initial temperature of the carrier gas. For all liquids, during an initial transient, the mean molar mass increases and the standard deviation of the PDF decreases due to evaporation of the lightest species; however, as the drops heat up and are moved by the flow, both evaporation and condensation occur, which increase both the mean and standard deviation of the PDF; the extent of these variations is liquid specific. The changes in the liquid composition induce variations in 
the gas composition. Volumetric averages show that the gas develops a very heterogeneous composition, and the single-peaked initial PDF is quickly and increasingly transformed into a double-peaked PDF. Separate volumetric averages over the lower stream and the mixing layer show that the double peak aspect may eventually subside in the lower stream due to saturation, whereas in the mixing layer the PDF becomes increasingly double-peaked.

Flow visualizations of multicomponent-liquid drop simulations display an inhomogeneous drop number density that is more complex in organization than that of the baseline single chemical-species drops simulation, and the multicomponentliquid drop visualizations exhibit a maximum positive spanwise vorticity magnitude that is liquid-specific. Analysis of the results shows that the small-scale vorticity production is reduced in simulations with diesel compared to n-decane, a feature which, based on examination of velocity-fluctuation spectra presented elsewhere, is attributed to the less effective energy cascade from the large to the small scales. Among multicomponent liquids, the positive spanwise vorticity and the small-scale activity increase with increasingly smaller-molar-mass species present in the initial liquid composition. An assessment of the homogeneous-plane-average budgets of the vorticity equation indicates that the stretching and tilting effect dominates the vorticity budgets, followed by the viscous stresses and by the momentum source term, with the other contributions being minimal. The momentum-source term exceeds the other two significant terms at locations in the upper part of the layer where the drop number density is largest in the field. The momentum-source term has potential contributions from drop drag, evaporation, and liquid mean molar mass change, although for the conditions of the present simulations the drag is the most effective term. It is conceivable that under conditions where the composition changes faster than the flow characteristic time compared to the present simulations, the evaporation and/or the mean molar mass change may dominate the momentum-source term, and thus the coupling between composition and dynamic flow features will be stronger.

Visualizations of the vapour mass fraction show that its maximum increases with liquid volatility and initial carrier gas temperature. For multicomponent liquids, the mean vapour molar mass decreases and the standard deviation increases with increased value of the initial vapour molar fraction in the gas owing to a reduced evaporation rate that prevents heavy species from leaving the liquid. With increased liquid volatility, both the maximum mean and the standard deviation of the vapour composition increase, resulting from the larger evaporation rate which permits the release of the high-molar-mass species once the low-molar-mass species have vaporized. Homogeneous-plane averages show strong composition differentiation among liquids and substantial specificity in their response to an increased carrier gas initial temperature. For all liquids, the composition PDF develops the largest second peak in the upper part of the layer at the locations of the highest drop number density, although for some liquids this second peak is minimal.

The conclusions are that strong composition non-uniformities in the layer, found for all multicomponent liquids, indicate that single-species liquids cannot capture the crucial physics of mixtures. Because the cascade of energy from the large to the small scales has been found to be reduced in some multicomponent-species layers, it is proposed that the turbulence development and its aspect may assume different characteristics according to the liquid. The gaseous-species distribution was found to be liquid-specific, offering the possibility of discriminating between the impact of different species on various phenomena. The simulations show that composition effects are amplified with increasing temperature. Likewise, for Reynolds numbers 
larger than that of the simulations, evaporation will be enhanced resulting again in amplified composition heterogeneity. Thus, caution must be exercised in extrapolating interpretation of experimental data obtained with single species to the more complex situation of liquids that are mixtures of many species.

This study was conducted at the Jet Propulsion Laboratory (JPL), California Institute of Technology (Caltech), under the partial sponsorship of the Donors of The Petroleum Research Fund administered by the American Chemical Society through a grant (to J. B.) for Caltech Post Doctoral Fellow support, the US Department of Energy with Ms R. Danz, Mr D. Hooker, Mr S. Waslo and Ms D. Haught as contract monitors, under an agreement with the National Aeronautics and Space Administration, and the Air Force Research Laboratory, Wright Patterson Air Force Base with Dr J.T. Edwards as Technical Monitor. Additional sponsorship was provided by the Office National d'Études et de Recherches Aérospatiales for Post Doctoral support. Computational resources were provided by the supercomputing facility at JPL.

\section{Appendix A. Properties used in the simulations}

In the simulations, air is treated as a single-pseudo-component inert carrier gas with an effective critical temperature $T_{c r}=133 \mathrm{~K}, m_{a}=29 \mathrm{~kg} \mathrm{kmol}^{-1}$ and ratio of heat capacities $\gamma_{\text {ther }, a}=1.4$. Then, $\lambda_{a}=1.36 \times 10^{-2}\left(T / T_{c}\right)^{0.75}\left(\mathrm{~W} \mathrm{mK}^{-1}\right)$ and $C_{p, a}=\gamma_{\text {ther, }} R_{u} /$ $\left(\gamma_{\text {ther }, a}-1\right) m_{a}\left(\mathrm{~kJ} \mathrm{kgK}^{-1}\right)$ where $R_{u}=8.3142\left(\mathrm{~kJ} \mathrm{kmolK}^{-1}\right)$.

Information from the American Petroleum Institute (1992) was used to obtain the correlation $T_{b}(m)=241.4+1.45 m(\mathrm{~K})$ and to develop the correlation

$$
\lambda_{v}(m)=3.45 \times 10^{-3} m^{0.37}\left(\frac{T}{T_{b}}\right)^{n}\left(\mathrm{~W} \mathrm{mK}^{-1}\right) \quad \text { where } \quad n=\frac{2.225 m}{m+19.245} .
$$

The correlations for the heat capacities were those of Tamim \& Hallett (1995), namely

$$
C_{p}(m)=\left(A_{p}+B_{p} m\right) R_{u} / m \quad\left(\mathrm{~kJ} \mathrm{kgK}^{-1}\right),
$$

where $A_{p}=2.465-0.1144 \times 10^{-2} T_{r}+1.759 \times 10^{-5} T_{r}^{2}-5.972 \times 10^{-9} T_{r}^{3}$ and $B_{p}=$ $-0.03561+9.367 \times 10^{-4} T_{r}-6.030 \times 10^{-7} T_{r}^{2}+1.324 \times 10^{-10} T_{r}^{3}$ with the reduced temperature $T_{r}=T / T_{c r}$. Further, two average heat capacities are defined

$$
\begin{aligned}
& C_{p, v}=X_{v} \int_{\gamma}^{\infty} C_{p}(m) P_{v}(m) \mathrm{d} m+\left(1-X_{v}\right) C_{p, a} \quad\left(\mathrm{~kJ} \mathrm{kmolK}^{-1}\right), \\
& \overline{C_{p, g}}=\left(1-X_{v}\right) C_{p a}+\left(A_{p}+B_{p} \theta_{v}\right) X_{v} R_{u} \quad\left(\mathrm{~kJ} \mathrm{kmolK}^{-1}\right) .
\end{aligned}
$$

Then, a ratio of heat capacities is defined

$$
\gamma_{\text {ther }, g}=\frac{\overline{C_{p, g}}}{\overline{C_{V, g}}} \text { with } \overline{C_{V, g}}=\overline{C_{p, g}}-R_{u},
$$

where $C_{V}$ is the heat capacity at constant volume. Both $\lambda_{v}(m)$ and $C_{p}(m)$, were calculated at the drop surface conditions, that is using $T_{d}$ and $m_{v}^{(s)}$.

An effective latent heat is defined as

$$
\begin{aligned}
L_{v e f f}= & \frac{1}{m^{(s)}}\left[\left(m^{(s)}-X_{v}^{(s)} \theta_{v}^{(s)}\right)\left(C_{p, a}-C_{l}\right) T_{d}+X_{v}^{(s)} \Delta s_{\mathrm{lg}}\left(A_{b}+B_{b} \theta_{v}^{(s)}\right)\right](1+B) \\
& -\frac{1}{m}\left[\left(m-X_{v} \theta_{v}\right)\left(C_{p, a}-C_{l}\right) T_{d}+X_{v} \Delta s_{\mathrm{lg}}\left(A_{b}+B_{b} \theta_{v}\right)\right]
\end{aligned}
$$


and following Tamim \& Hallett (1995), $C_{l}=2.26-2.94 \times 10^{-3} T_{r, d}+9.46 \times 10^{-6} T_{r, d}^{2}$ $\left(\mathrm{kJ} \mathrm{kgK}^{-1}\right)$.

According to Miller, Harstad \& Bellan (1998),

$$
\begin{aligned}
P r & =0.815-4.958 \times 10^{-4} T_{r e f}+4.515 \times 10^{-7} T_{r e f}^{2}, \\
T_{r e f} & =(2 / 3) T_{d}+(1 / 3) T,
\end{aligned}
$$

leading to $\operatorname{Pr}$ values of $0.696,0.694$ and 0.693 for $T=375 \mathrm{~K}, 400 \mathrm{~K}$ and $425 \mathrm{~K}$, respectively.

Properties for a mixture of species were calculated using mixing rules.

\section{Appendix B. Characteristic boundary conditions for the double Gamma PDF formulation}

The rigorous method of Poinsot \& Lele (1992), called Navier-Stokes characteristic boundary conditions (NSCBC), was implemented here for the DGPDF in the context of the local one-dimensional inviscid (LODI) framework in order to mitigate the lack of physical boundary conditions in the $x_{2}$-direction for determining all primitive variables. The CT-modified Navier-Stokes system of equations is written as

$$
\frac{\partial \widetilde{\boldsymbol{U}}}{\partial t}+\frac{\partial \boldsymbol{F}^{(k)}}{\partial x_{j}}+\widetilde{\boldsymbol{C}}^{(k)}=0
$$

where the set of conservative variables is defined by

$$
\widetilde{\boldsymbol{U}}=\left(c, c m u_{j}, c m e_{t}, c X_{v}, c X_{v} \theta_{v}, c X_{v} \psi_{v}, c X_{v} \xi_{3 v}, c X_{v} \xi_{4 v}\right),
$$

the flux vector of the conservative variables is

$$
\boldsymbol{F}^{(k)}=\left[c u_{j}, c u_{j} u_{k},\left(c m e_{t}+p\right) u_{j}, c X_{v} u_{j}, c X_{v} \theta_{v} u_{j}, c X_{v} \psi_{v} u_{j}, c X_{v} \xi_{3 v} u_{j}, c X_{v} \xi_{4 v} u_{j}\right]
$$

for integer $k \in[1,3]$, and $\widetilde{\boldsymbol{C}}^{(k)}$ includes all terms without any first derivative of $\widetilde{\boldsymbol{U}}$ along the $j$-direction, which are considered to be passively convected along the $j$-direction without modifying the present characteristic wave analysis. Thus,

$$
\begin{aligned}
\widetilde{\boldsymbol{C}}^{(k)}= & \left\{-\frac{\partial}{\partial x_{j}}\left[c \mathscr{D} \frac{\partial}{\partial x_{j}}\left(X_{v}\right)\right]+\frac{1}{m_{a}} \frac{\partial}{\partial x_{j}}\left[c \mathscr{D} \frac{\partial}{\partial x_{j}}\left(X_{v} \theta_{v}\right)\right]-S_{\text {mole }},-\frac{\partial \tau_{k j}}{\partial x_{j}}-S_{\text {mom }, k},\right. \\
& -\frac{\partial}{\partial x_{j}}\left[\lambda \frac{\partial T_{g}}{\partial x_{j}}+u_{i} \tau_{i j}-\sum_{\zeta=[a,[1, N]]} J_{D \zeta, j} h_{\zeta}\right]-S_{\mathrm{en}},-\frac{\partial}{\partial x_{j}}\left[c \mathscr{D} \frac{\partial X_{v}}{\partial x_{j}}\right]-S_{\text {mole }} \\
& -\frac{\partial}{\partial x_{j}}\left[c \mathscr{D} \frac{\partial}{\partial x_{j}}\left(X_{v} \theta_{v}\right)\right]-S_{\text {mass }},-\frac{\partial}{\partial x_{j}}\left[c \mathscr{D} \frac{\partial}{\partial x_{j}}\left(X_{v} \psi_{v}\right)\right]-S_{\psi}, \\
& \left.-\frac{\partial}{\partial x_{j}}\left[c \mathscr{D} \frac{\partial}{\partial x_{j}}\left(X_{v} \xi_{v 3}\right)\right]-S_{\xi_{3}},-\frac{\partial}{\partial x_{j}}\left[c \mathscr{D} \frac{\partial}{\partial x_{j}}\left(X_{v} \xi_{v 4}\right)\right]-S_{\xi_{4}}\right\} .
\end{aligned}
$$

The system of equations is closed by using the perfect gas law $p=c R_{u} T$ for the mixture. In order to identify characteristic waves in the hyperbolic part of the CTmodified system of equations, an equivalent form of the system using the primitive variables $\boldsymbol{U}=\left(c, u_{1}, u_{2}, u_{3}, p, X_{v}, \theta_{v}, \psi_{v}, \xi_{3 v}, \xi_{4 v}\right)$ was derived. If $\boldsymbol{P}$ is the matrix relating the primitive variables to the conservative variables

$$
\boldsymbol{P}=\frac{\partial \widetilde{\boldsymbol{U}}}{\partial \boldsymbol{U}},
$$




$$
\boldsymbol{P}=\left(\begin{array}{cccccccccc}
1 & 0 & 0 & 0 & 0 & 0 & 0 & 0 & 0 & 0 \\
m u_{1} & m c & 0 & 0 & 0 & c u_{1}\left(\theta_{v}-m_{a}\right) & c u_{1} X_{v} & 0 & 0 & 0 \\
m u_{2} & 0 & m c & 0 & 0 & c u_{2}\left(\theta_{v}-m_{a}\right) & c u_{2} X_{v} & 0 & 0 & 0 \\
m u_{3} & 0 & 0 & m c & 0 & c u_{3}\left(\theta_{v}-m_{a}\right) & c u_{3} X_{v} & 0 & 0 & 0 \\
P_{5,1} & c u_{1} & c u_{2} & c u_{3} & \frac{1}{\gamma_{\text {ther, }}-1} & P_{5,6} & P_{5,7} & P_{5,8} & 0 & 0 \\
X_{v} & 0 & 0 & 0 & 0 & c & 0 & 0 & 0 & 0 \\
X_{v} \theta_{v} & 0 & 0 & 0 & 0 & c \theta_{v} & c X_{v} & 0 & 0 & 0 \\
X_{v} \psi_{v} & 0 & 0 & 0 & 0 & c \psi_{v} & 0 & c X_{v} & 0 & 0 \\
X_{v} \xi_{3 v} & 0 & 0 & 0 & 0 & c \xi_{3 v} & 0 & 0 & c X_{v} & 0 \\
X_{v} \xi_{4 v} & 0 & 0 & 0 & 0 & c \xi_{4 v} & 0 & 0 & 0 & c X_{v}
\end{array}\right),
$$

where $\gamma_{\text {ther,g }}$ is the ratio of the constant pressure and constant volume specific heats computed as in Appendix A

$$
\begin{gathered}
P_{5,1}=m \frac{u_{i} u_{i}}{2}+X_{v}\left(-R_{u} A_{p} A_{b}+\Delta s_{\mathrm{lg}} A_{b}+C_{l} A_{b}\right) \\
+X_{v} \theta_{v}\left(-R_{u} B_{p} A_{b}+\Delta s_{\mathrm{lg}} B_{b}+C_{l} B_{b}-R_{u} B_{b} A_{p}\right)-X_{v} \psi_{v} R_{u} B_{b} B_{p}, \\
P_{5,6}=p\left(A_{p}+\theta_{v} B_{p}-\frac{C_{p a}}{R_{u}}\right)-c R_{u} A_{p} A_{b}+c \Delta s_{\mathrm{lg}} A_{b}+c C_{l} A_{b}-c \theta_{v} R_{u} B_{p} A_{b} \\
+c \theta_{v} \Delta s_{\mathrm{lg}} B_{b}+c C_{l} B_{b} \theta_{v}-c \theta_{v} R_{u} B_{b} A_{p}-c \psi_{v} R_{u} B_{b} B_{p}+\left(\theta_{v}-m_{a}\right)\left(\frac{c u_{i} u_{i}}{2}\right), \\
P_{5,7}=X_{v} B_{p} p-c X_{v} R_{u} B_{p} A_{b}+c X_{v} \Delta s_{\mathrm{lg}} B_{b}+c X_{v} C_{l} B_{b}-c X_{v} R_{u} B_{b} A_{p}+c X_{v} \frac{u_{i} u_{i}}{2}, \\
P_{5,8}=-c X_{v} R_{u} B_{p} B_{b},
\end{gathered}
$$

with $C_{l}$ and $C_{p a}$ calculated as in Appendix A.

The wave amplitudes corresponding to each eigenvalue $\kappa_{i}$ are

$$
\left.\begin{array}{l}
\mathscr{L}_{1}=\left(u_{2}-a\right)\left[\frac{\partial p}{\partial x_{2}}-m c a \frac{\partial u_{2}}{\partial x_{2}}\right] \quad \text { for } \kappa_{1}=u_{2}-a, \\
\mathscr{L}_{2}=u_{2}\left[\frac{\partial p}{\partial x_{2}}-m a^{2} \frac{\partial c}{\partial x_{2}}\right] \quad \text { for } \kappa_{2}=u_{2}, \\
\mathscr{L}_{3}=u_{2} \frac{\partial u_{1}}{\partial x_{2}}, \mathscr{L}_{4}=u_{2} \frac{\partial u_{3}}{\partial x_{2}} \quad \text { for } \kappa_{i}=u_{2}, i=3,4, \\
\mathscr{L}_{5}=u_{2} \frac{\partial X_{v}}{\partial x_{2}}, \mathscr{L}_{6}=u_{2} \frac{\partial \theta_{v}}{\partial x_{2}}, \mathscr{L}_{7}=u_{2} \frac{\partial \psi_{v}}{\partial x_{2}} \quad \text { for } \kappa_{i}=u_{2}, i=5,6,7, \\
\mathscr{L}_{8}=u_{2} \frac{\partial \xi_{3 v}}{\partial x_{2}}, \mathscr{L}_{9}=u_{2} \frac{\partial \xi_{4 v}}{\partial x_{2}} \quad \text { for } \kappa_{i}=u_{2}, i=8,9, \\
\mathscr{L}_{10}=\left(u_{2}+a\right)\left[\frac{\partial p}{\partial x_{2}}+m c a \frac{\partial u_{2}}{\partial x_{2}}\right] \quad \text { for } \kappa_{10}=u_{2}+a .
\end{array}\right\}
$$

The cross-stream boundaries are adiabatic slip walls. There is one inviscid condition to satisfy on the slip wall, $u_{2}=0$; the viscous relations are the null tangential stresses $\tau_{12}=\tau_{23}=0$; and the heat flux through the adiabatic wall is null,

$$
\lambda \frac{\partial T}{\partial y}+v \tau_{2 j}-\sum_{\zeta=[a,[1, N]]} J_{D \zeta, 2} h_{\zeta}=0 .
$$


Since the normal velocity is null, the amplitudes $\mathscr{L}_{2}$ to $\mathscr{L}_{7}$ are null and $\mathscr{L}_{1}=\mathscr{L}_{10}$. The amplitude of the reflected wave $\mathscr{L}_{1}$ is thus set to $\mathscr{L}_{10}$, with $\mathscr{L}_{10}$ computed from the variables inside the domain.

\section{REFERENCES}

American Petroleu Institute 1992 Technical Data Book - Petroleum Refining, 5th Edn. American Petroleum Institute.

Arias-Zugasti, M. \& Rosner, D. E. 2003 Multicomponent fuel droplet vaporization and combustion using spectral theory for a continuous mixture. Combust. Flame 135, 271-284.

Batchelor, G. K. 1967 The Theory of Homogeneous Turbulence. Cambridge University Press.

Baum, M., Poinsot, T. J. \& Lele, S. K. 1994 Accurate boundary conditions for multicomponent reactive flows. J. Comput. Phys. 116, 247-261.

BoIvin, M., SimOnin, S. \& SQuires, K. D. 1988 Direct numerical simulation of turbulence modulation by particles in isotropic turbulence. J. Fluid Mech. 375, 235-263.

Chorin, A. J. 1991 Vorticity and Turbulence. Springer.

Chou, G. F. \& Prausnitz, J. M. 1986 Adiabatic flash calculations for continuous or semicontinuous mixtures using an equation of state. Fluid Phase Equilibria 30, 75-82.

Continillo, G. \& Sirignano, W. A. 1988 Numerical study of multicomponent fuel spray flame propagation in a spherical closed volume. Proc. Combust. Inst. 22, 1941-1949.

Cotterman, R. L., Bender, R. \& Prausnitz, J. M. 1985 Phase equilibria for mixtures containing very many components. Development and application of continuous thermodynamics for chemical process design. Ind. Engng Chem. Process Des. Dev. 24, 194-203.

Edwards, T. \& MaURice, L. Q. 2001 Surrogate mixtures to represent complex aviation and rocket fuels. J. Propul. Power 17(2), 461-466.

Gal-Or, B., Cullinan, JR., H. T. \& Galli, R. 1975 New thermodynamic-transport theory for systems with continuous component density distributions. Chem. Engng Sci. 30, 1085-1092.

Hallett, W. L. H. 2000 A simple model for the vaporization of droplets with large numbers of components. Combust. Flame 121, 334-344.

HARSTAD, K. 2005 Trend analysis for atmospheric hydrocarbon partitioning using continuous thermodynamics J. Atmos. Sci. (accepted).

Harstad, K. \& Bellan, J. 1991 A Model of the evaporation of binary-fuel clusters of drops. Atomiz. Sprays 1, 367-388.

Harstad, K. \& Bellan, J. 2004 Modeling evaporation of Jet A, JP-7 and RP-1 drops at 1 to 15 bars. Combust. Flame 137, 163-177.

Harstad, K. G., Le ClercQ, P. C. \& Bellan, J. 2003 A statistical model of multicomponent-fuel drop evaporation for many-droplet gas-liquid flow simulations. AIAA J. 41, 1858-1874.

Kennedy, C. \& CARpenter, M. 1994 Several new numerical methods for compressible shear layer simulations. Appl. Numer. Maths 14, 397-433.

LAw, C. K. \& LAW, H. K. 1982 A $d^{2}$-law for multicomponent droplets vaporization and combustion. AIAA J. 20, 522-527.

Le ClercQ, P. C. \& Bellan, J. 2004 Direct numerical simulation of a transitional temporal mixing layer laden with multicomponent-fuel evaporating drops using continuous thermodynamics. Phys. Fluids 16, 1884-1907.

LiPPERT, A. M. \& ReITZ, R. D. 1997 Modeling of multicomponent fuels using continuous distributions with application to droplet evaporation and sprays. SAE Paper 97FL-468.

MaKino, A. \& LAW, C. K. 1988 On the controlling parameter in the gasification behaviour of multicomponent droplets. Combust. Flame 73, 331-336.

MashaYeK, F. 1998 a Droplet-turbulence interactions in low-Mach-number homogeneous shear two-phase flows. J. Fluid Mech. 367, 163-203.

MashaYeK, F. 1998 a Direct numerical simulations of evaporating droplet dispersion in forced low-Mach-number turbulence. Intl J. Heat Mass Transfer 41, 2601-2617.

MashaYeK, F. \& JABERI, F. A. 1999 Particle dispersion in forced isotropic low-Mach-number turbulence. Intl J. Heat Mass Transfer 42, 2823-2836.

Megaridis, C. M. \& Sirignano, W. A. 1990 Numerical modeling of a vaporizing multicomponent droplet. Proc. Combust. Inst. 23, 1413-1421. 
Miller, R. S. \& Bellan, J. 1999 Direct numerical simulation of a confined three-dimensional gas mixing layer with one evaporating hydrocarbon-droplet laden stream. J. Fluid Mech. 384, 293-338.

Miller, R. S. \& Bellan, J. 2000 Direct numerical simulation and subgrid analysis of a transitional droplet laden mixing layer. Phys. Fluids 12, 650-671.

Miller, R. S., Harstad, K. \& Bellan, J. 1998 Evaluation of equilibrium and non-equilibrium evaporation models for many-droplet gas-liquid flow simulations. Intl J. Multiphase Flow 24, $1025-1055$.

Moser, R. D. \& Rogers, M. M. 1991 Mixing transition and the cascade to small scales in a plane mixing layer. Phys. Fluids A 3, 1128-1134.

Okong'o, N. A. \& Bellan, J. 2004 Consistent large eddy simulation of a temporal mixing layer laden with evaporating drops. Part 1. Direct numerical simulation, formulation and a priori analysis. J. Fluid Mech. 499, 1-47.

Poinsot, T. J. \& Lele, S. K. 1992 Boundary conditions for direct simulations of compressible viscous flows. J. Comput. Phys. 101, 104-129.

Rätzsch, M. T. \& Kehlen, H. 1983 Continuous thermodynamics model of complex mixtures. Fluid Phase Equilibria 14, 225-234.

RÉVEILlON, J. \& VERVISCH, L. 2000 Spray vaporization in nonpremixed turbulent combustion modeling: A single drop model. Combust. Flame 121, 75-90.

Squires, K. D. \& Eaton, J. K. 1991 Preferential concentration of particles by turbulence. Phys. Fluids A 3, 1169-1178.

Tamim, J. \& Hallett, W. L. H. 1995 A continuous thermodynamics model for multicomponent droplet vaporization. Chem. Engng Sci. 50, 2933-2942.

Tennekes, H. \& Lumley, J. L. 1972 A First Course in Turbulence. MIT Press.

Whitson, C. H. 1983 Characterizing hydrocarbon plus fractions. Soc. Petrol Engng J. 23, 683-694.

Williams, F. A. 1965 Combustion Theory. Addison-Wesley.

Zheng, Y. \& LeE, C. F. 2002 A preferential vaporization model for multicomponent droplets and sprays. Atomiz. Sprays 12, 163-186. 